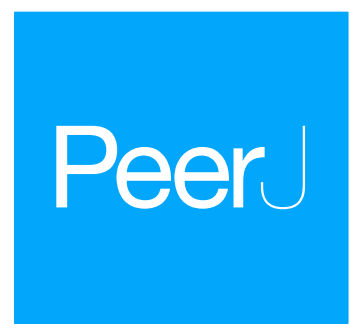

Submitted 19 February 2018

Accepted 28 March 2018

Published 16 April 2018

Corresponding author

Sergey Y. Morozov,

morozov@genebee.msu.su

Academic editor

Yegor Vassetzky

Additional Information and Declarations can be found on page 19

DOI 10.7717/peerj.4636

Copyright

2018 Morozov et al.

Distributed under

Creative Commons CC-BY 4.0

OPEN ACCESS

\section{TAS3 miR390-dependent loci in non-vascular land plants: towards a comprehensive reconstruction of the gene evolutionary history}

\author{
Sergey Y. Morozov ${ }^{1}$, Irina A. Milyutina ${ }^{1}$, Tatiana N. Erokhina ${ }^{2}$, \\ Liudmila V. Ozerova ${ }^{3}$, Alexey V. Troitsky ${ }^{1}$ and Andrey G. Solovyev ${ }^{1,4}$ \\ ${ }^{1}$ Belozersky Institute of Physico-Chemical Biology, Moscow State University, Moscow, Russia \\ ${ }^{2}$ Shemyakin-Ovchinnikov Institute of Bioorganic Chemistry, Russian Academy of Science, Moscow, Russia \\ ${ }^{3}$ Tsitsin Main Botanical Garden, Russian Academy of Science, Moscow, Russia \\ ${ }^{4}$ Institute of Molecular Medicine, Sechenov First Moscow State Medical University, Moscow, Russia
}

\section{ABSTRACT}

Trans-acting small interfering RNAs (ta-siRNAs) are transcribed from protein noncoding genomic TAS loci and belong to a plant-specific class of endogenous small RNAs. These siRNAs have been found to regulate gene expression in most taxa including seed plants, gymnosperms, ferns and mosses. In this study, bioinformatic and experimental PCR-based approaches were used as tools to analyze TAS3 and TAS6 loci in transcriptomes and genomic DNAs from representatives of evolutionary distant non-vascular plant taxa such as Bryophyta, Marchantiophyta and Anthocerotophyta. We revealed previously undiscovered TAS3 loci in plant classes Sphagnopsida and Anthocerotopsida, as well as TAS6 loci in Bryophyta classes Tetraphidiopsida, Polytrichopsida, Andreaeopsida and Takakiopsida. These data further unveil the evolutionary pathway of the miR390-dependent TAS3 loci in land plants. We also identified charophyte alga sequences coding for SUPPRESSOR OF GENE SILENCING 3 (SGS3), which is required for generation of ta-siRNAs in plants, and hypothesized that the appearance of TAS3-related sequences could take place at a very early step in evolutionary transition from charophyte algae to an earliest common ancestor of land plants.

Subjects Bioinformatics, Genomics, Molecular Biology, Plant Science

Keywords Silencing, Trans-acting RNA, Small interfering RNA, ARF genes, Micro RNA, Charophyte algae, Bryophytes

\section{INTRODUCTION}

Plant chromosomal loci of trans-acting small interfering RNAs (ta-siRNAs) and microRNAs (miRNAs) encode non-protein-coding and protein-coding precursor transcripts, which are synthesized by RNA polymerase II and include cap-structures and poly-(A) tails. In plants, primary miRNA transcripts forming internal imperfect hairpins are processed by a protein complex including Dicer-like protein 1 (DCL1), HYL1 and SERRATE to give RNA duplexes with 2-nucleotide 3 '-overhangs, which are then terminally methylated by specific RNA methylase HEN1. One strand of such duplexes, being typically of 21 nucleotides in 
length and representing a mature miRNA, is selectively recruited by Argonaut (AGO) family protein to an effector complex targeting a specific RNA for AGO-mediated endonucleolytic cleavage or translational repression (Rogers \& Chen, 2013; Axtell, 2013; Bologna \& Voinnet, 2014; Borges \& Martienssen, 2015; Chorostecki et al., 2017).

Some specific microRNAs are able to initiate production of ta-siRNAs (and other secondary phased RNAs_-phasiRNAs) by an step-by-step processing of long doublestranded RNA by DCL4 from a start point defined by miRNA-directed cleavage of a single-stranded RNA precursor in a "phased" pattern. These PHAS loci include noncoding TAS genes and genes encoding penta-tricopeptide repeat-containing proteins (PPRs), nucleotide-binding and leucine-rich repeat-containing proteins (NB-LRRs), or MYB transcription factors (Allen \& Howell, 2010; Zhai et al., 2011; Xia et al., 2013; Fei, Xia \& Meyers, 2013; Axtell, 2013; Yoshikawa, 2013; Zheng et al., 2015; Komiya, 2017; Liu et al., 2018; Deng et al., 2018). Biogenesis of ta-siRNAs includes initial AGO-dependent miRNA binding at single or dual sites of the precursor transcripts and their subsequent cleavage. The further process is dependent on plant RNA-dependent RNA polymerase 6 (RDR6) and SGS3 proteins participating in the formation of dsRNA, which is then cleaved in a sequential and phased manner by DCL4 with assistance of DRB4 (dsRNA binding protein). The resulting ta-siRNAs (mostly of $21 \mathrm{bp}$ in length), similar to miRNAs, are methylated by HEN1 protein (Allen \& Howell, 2010; Axtell, 2013; Fei, Xia \& Meyers, 2013; Yoshikawa, 2013; Bologna \& Voinnet, 2014; Komiya, 2017; Deng et al., 2018).

Arabidopsis TAS3a transcript, first identified by Allen et al. (2005), gives rise to two near-identical 21-nucleotide tasiARFs targeting the mRNAs of some auxin-responsive transcription factors (ARF2, ARF3/ETT and ARF4). Most angiosperm TAS3 primary transcripts are recognized by miR390 and cleaved by AGO7 at the $3^{\prime}$ target site, whereas the $5^{\prime}$ miRNA target site is non-cleavable. However, the number of miR390 cleavage sites, organization of tasiARF sequence blocks and phasing registers may vary among different TAS3 genes of vascular plants (Allen \& Howell, 2010; Axtell, 2013; Fei, Xia \& Meyers, 2013; Zheng et al., 2015; Xia et al., 2013; Xia, Xu \& Meyers, 2017; De Felippes et al., 2017; Komiya, 2017; Deng et al., 2018). Moreover, miR390 may additionally target and inhibit protein-coding gene transcripts, such as StCDPK1 related to auxin-responsive pathway (Santin et al., 2017).

Previously, we described a new method for identification of plant TAS3 loci based on PCR with a pair of oligodeoxyribonucleotide primers mimicking miR390. The method was found to be efficient for dicotyledonous plants, cycads, conifers, and mosses (Krasnikova et al., 2009; Krasnikova et al., 2011; Krasnikova et al., 2013; Ozerova et al., 2013). Importantly, at that time the structural and functional information on bryophyte TAS3 loci was available only for the model plant Physcomitrella patens (Arif, Frank \& Khraiwesh, 2013), and we used our PCR-based approach as a phylogenetic profiling tool to identify relatives of $P$. patens TAS3 loci in 26 additional moss species of class Bryopsida and several mosses of classes Polytrichopsida, Tetraphidopsida and Andreaeopsida. Moreover, we found a putative pre-miR390 genomic sequence for an additional moss class, Oedipodipsida (Krasnikova et al., 2013). Our studies revealed that a representative of Marchantiophyta (liverwort Marchantia polymorpha, class Marchantiopsida) could also encode a candidate miR390 gene 
and a potential TAS3-like locus (Krasnikova et al., 2013). This finding extended the known evolutionary history of TAS3 loci to the proposed most basal land plant lineage (Ruhfel et al., 2014; Bowman et al., 2017). In addition, we sequenced putative pre-miR390 genomic locus for Harpanthus flotovianus (Marchantiophyta, class Jungermanniopsida) (Krasnikova et al., 2013). Later, our findings of TAS3-like and miR390 loci were experimentally confirmed in the studies of the transcriptomes of Marchantiophyta plants M. polymorpha (Lin et al., 2016; Tsuzuki et al., 2016) and Pellia endiviifolia (class Jungermanniopsida) (Alaba et al., 2015).

New genomic and transcriptomic sequence data for basal Viridiplantae appeared in NCBI (http://ncbi.nlm.nih.gov/sra) and Phytozome (http://www.phytozome.net) databases prompted us to perform new experimental and in silico analyses of TAS3 loci in basal taxons of Viridiplantae. In this paper, we identified previously unrecognized TAS3 loci in classes Sphagnopsida and Anthocerotopsida, as well as composite TAS6/TAS3 loci in classes Tetraphidiopsida, Polytrichopsida, Andreaeopsida and Takakiopsida. Additionally, we revealed SGS3-coding sequences in charophytes and analyzed their evolutionary links.

\section{MATERIALS AND METHODS}

Dried material for Sphagnum angustifolium and S. girgensohnii were taken from herbarium at Department of Biology, Moscow State University. Total DNA was extracted from dry plants using the Nucleospin Plant Extraction Kit (MachereyNagel, Düren, Germany) according to the protocol of the manufacturer. For PCR amplification, the following degenerate primers were used: a forward primer SphaTASP (5'-GGCGRTAWCCYTACTGAGCTA- $\left.3^{\prime}\right)$ and reverse primer Spha-TASM (5'TAGCTCAGGAGRGATAMMBMRA-3'). For PCR, 30 cycles were used with a melting temperature of $94^{\circ} \mathrm{C}-3^{\prime}$, and the next steps are as follows: an annealing temperature $94{ }^{\circ} \mathrm{C}-20^{\prime \prime}, 65^{\circ} \mathrm{C}-20^{\prime \prime}, 58^{\circ} \mathrm{C}-30^{\prime \prime}$, and an extending temperature of $72{ }^{\circ} \mathrm{C}$ followed by a final extension at $72{ }^{\circ} \mathrm{C}$ for $5^{\prime}$. PCR products were separated by electrophoresis of samples in a $1.5 \%$ agarose gel and purified using the Gel Extraction Kit (Qiagen, Hilden, Germany). For cloning, the PCR-amplified DNA bands isolated from gel were ligated into pGEM-T (Promega, Madison, WI, USA). The resulting clones were screened by length in $1,5 \%$ agarose gel. The plasmids were used as templates in sequencing reactions with an automated sequencer (Applied Biosystems, Foster City, CA, USA) 3730 DNA Analyzer with facilities of "Genom" (Moscow, Russia).

Sequences for comparative analysis were retrieved from NCBI (http://www.ncbi.nlm. nih.gov/), Phytozome (http://www.phytozome.net) and 1,000 Plant Transcriptome Project (“1KP”) (http://1kp-project.com/blast.html). Sequence similarities were analysed by NCBI Blast at http://blast.ncbi.nlm.nih.gov/BlastAlign.cgi. The presence of open reading frames within retrieved sequences was analysed at http://web.expasy.org/translate/. The nucleic acid sequences and deduced amino acid sequences were analyzed and assembled using the NCBI. Conserved domains in the amino acid sequences SGS3 were identified using the CD-Search of the NCBI.

The sequences of SGS3 protein and TAS3 nucleotide sequences were aligned by MAFFT version 7 software (Katoh \& Standley, 2014). The phylogenetic tree was constructed by the 
Neighbor-Joining method with 1,000 bootstrap replications in MEGA7 (Kumar, Stecher \& Tamura, 2016). The evolutionary distances were computed using the JTT matrix-based method and are in the units of the number of amino acid substitutions per site. The rate variation among sites was modeled with a gamma distribution (

\section{RESULTS}

\section{TAS3 loci in Bryophyta (classes Sphagnopsida and Takakiopsida)}

It is commonly accepted that mosses of classes Sphagnopsida and Takakiopsida represent most basal lineages in Bryophyta (Shaw et al., 2010; Shaw, Szövényi \& Shaw, 2011; Rosato et al., 2016). Previously, using primers, which have allowed us to detect pre-miR390 and TAS3 loci in Bryopsida and some other moss classes, we failed to identify pre-miR390 and TAS3 genes in genus Sphagnum (Krasnikova et al., 2013). However, a predicted sequence of pri-miR390 from Sphagnum fallax was recently reported (Xia, Xu \& Meyers, 2017). This finding prompted us to re-evaluate the occurrence of TAS3-like loci in Sphagnopsida. To this end, we designed a new pair of degenerated PCR primers Spha-TASP and Spha-TASM, which differed from those used previously (Krasnikova et al., 2011; Krasnikova et al., 2013). As a positive control, we used plasmid DNA carrying cloned TAS3 gene of Andreaea rupestris, a representative of basal Bryophyta (Krasnikova et al., 2013). Like the positive control, two total DNA probes from Sphagnum angustifolium and S. girgensohnii gave a single main PCR product of the expected size (Fig. 1). Cloning and sequencing of these PCR fragments revealed two TAS3-like primary structures having 285 (S. angustifolium) and 292 (S. girgensohnii) bases in length and exhibiting 96\% identity ( $e$-value $=2 \mathrm{e}-131$ ). We named these loci as Sphan-285 and Sphgi-292, (Fig. 2, Fig. S1 and Table 1). Despite that the degenerate miR390-mimicking primers were used for amplification of these loci, miR390 recognition sites in TAS3 species well corresponded to genomic loci of TAS3 extracted from genomic and transcriptomic data of other plants from genus Sphagnum (Fig. 2).

Peatmosses S. angustifolium and S. girgensohnii belong to subgenera Cuspidata and Acutifolia, respectively (Shaw et al., 2010; Shaw et al., 2016). To extend search for TAS3like loci inside genus Sphagnum we performed bioinformatics analysis of the nucleotide sequences in databases available at NCBI (Sequence Read Archive) and Phytozome (version 12.1). Phytozome has recently released genome assembly of bog moss S. fallax (version 0.5). Bog moss belongs to subgenus Cuspidata and represents the most closely related moss to S. angustifolium (Shaw et al., 2016). BLASTN search at Phytozome allowed us to reveal a TAS3-like locus (supercontig super_37), which has $100 \%$ identity to the TAS3 locus of S. angustifolium sequenced in this study (Fig. S1 and Table 1). Unexpectedly, we found an additional TAS3-like locus in S. fallax (transcript Sphfalx0293s0011, supercontig super_293). This TAS3 locus in bog moss has 277 nucleotides in length and showed only a distant relation to the S. angustifolium TAS3 (Fig. 2, Fig. S1 and Table 1).

To further analyze Sphagnopsida TAS3-related loci, we used BLAST analysis of Sequence Read Archive (SRA), which is the NCBI database collecting sequence data obtained by the use of next generation sequence (NGS) technology. Assembly of sequence reads of S. recurvum (subgenus Cuspidata) retrieved by BLAST search using S.fallax sequences as 


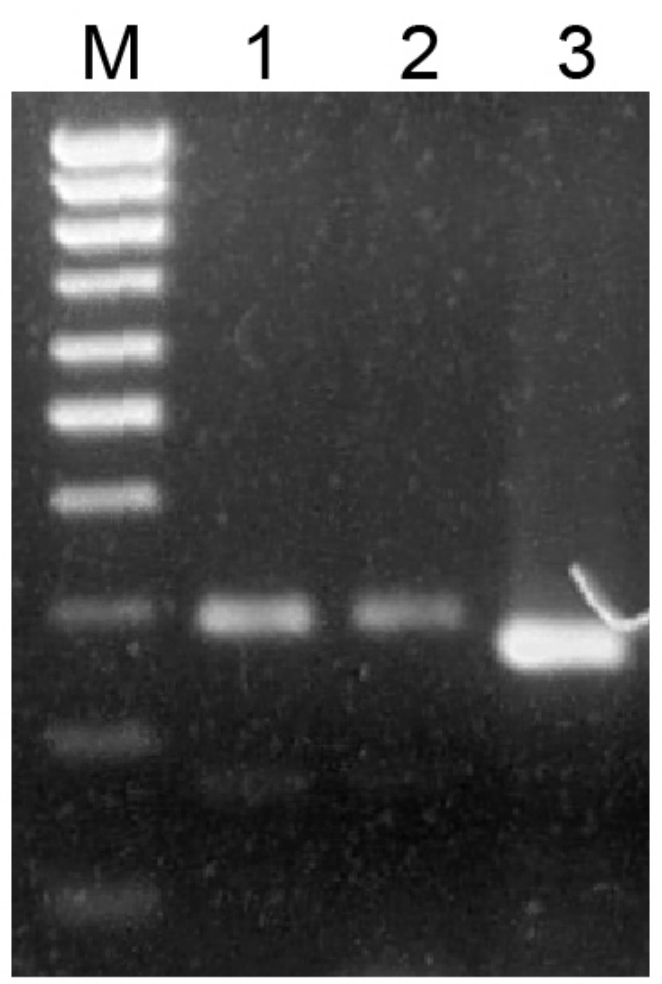

Figure 1 Analysis of PCR products in 1.5\% agarose gel. Amplification of genomic DNA sequences flanked by miR390 and miR390* sites. PCR products were obtained on genomic DNAs with degenerate primers. Sphagnum angustifolium (1), Sphagnum girgensohnii (2), Andreaea rupestris (3). (M), DNA size markers including bands ranging from $100 \mathrm{bp}$ to $1,000 \mathrm{bp}$ with $100 \mathrm{bp}$ step (Sibenzyme).

queries revealed two TAS3 loci (Table 1). The first locus (Sphre-283) is 283 nucleotides in length and has 98\% identity to Sphan-285. The second locus (Sphre-277) shows 98\% identity to Sphfalx0293s0011 (Table 1, Fig. S1). These findings indicate that two distant TAS3 loci in species of a particular subgenus of genus Sphagnum are extremely similar.

We also analyzed the SRA database of subgenus Sphagnum (Shaw et al., 2010; Shaw et al., 2016). It was found that $S$. magellanicum belonging to this subgenus also encode two TAS3 loci called Sphma-285 (285 nt size) and Sphma-286 (286 nt size) (Fig. S1 and Table 1). Unlike S. fallax and S. recurvum, in S. magellanicum TAS3 loci are more similar, showing 86\% identity (Fig. 2). Both Sphma-285 and Sphma-286 had 85\% identity to Sphan-285 (Fig. 2). It was found that TAS3-like locus (Sphpa) from one more representative of subgenus Sphagnum (S. palustre) exhibited 98\% identity to Sphma-285 (Fig. S1 and Table 1). The SRA database also contained sequence reads of two representatives from subgenus Subsecunda (Shaw et al., 2010; Shaw et al., 2016). Our BLAST analysis and subsequent assembly of retrieved reads revealed a single TAS3 locus in S. cribrosum (Sphcri, $291 \mathrm{nt}$ size) showing 95\% identity to Sphan-285 and 81\% identity to Sphma-286 (Fig. 2, Fig. S1 and Table 1) and a partial TAS3-like sequence in S. lescurii (Fig. S1 and Table 1).

Analysis of the SRA database of Takakia lepidozioides (class Takakiopsida) allowed us to reveal only one TAS3-like sequence (Takle-207) (Fig. S1 and Table 1). The same sequence 
(A)

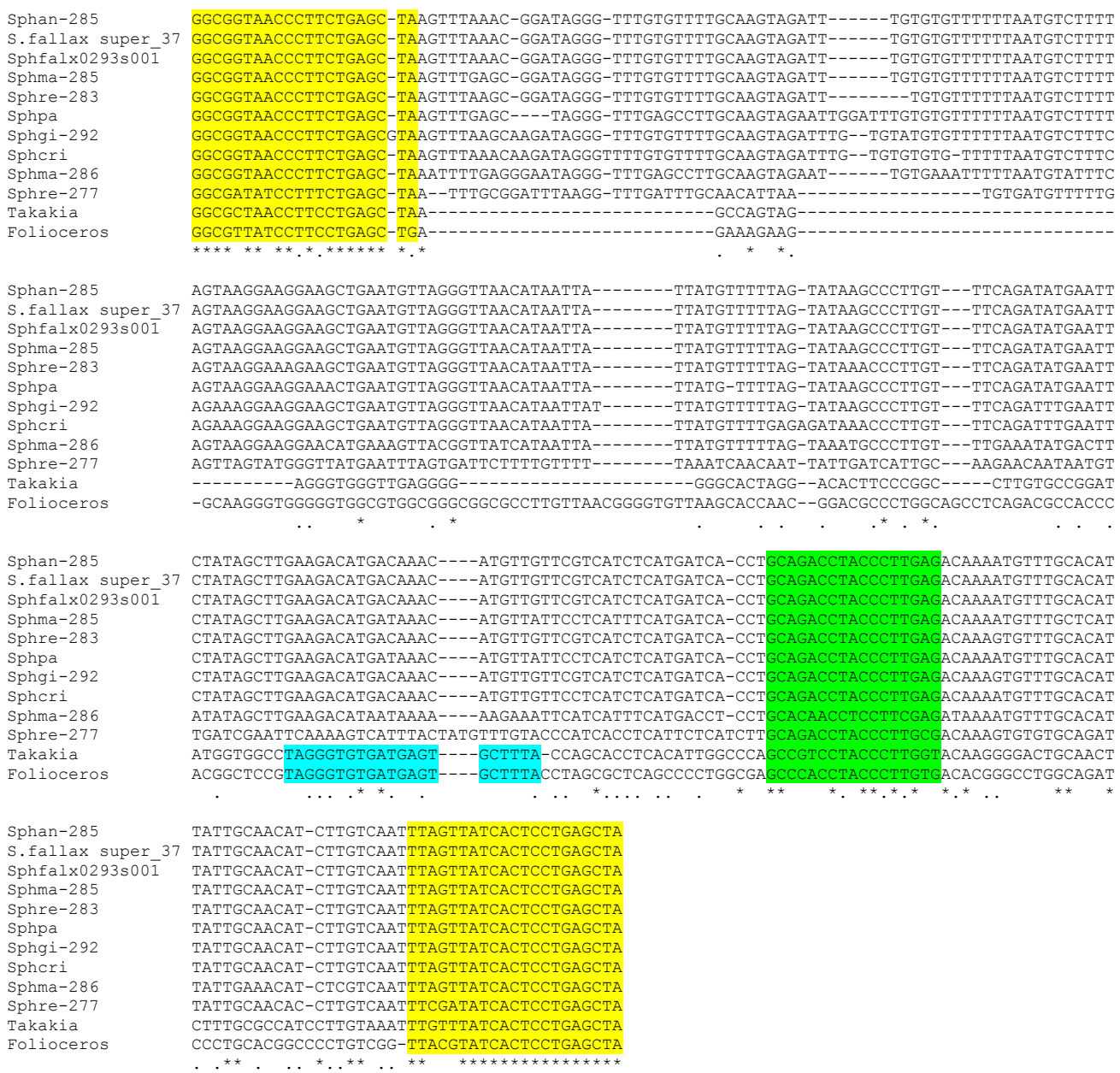

(B)

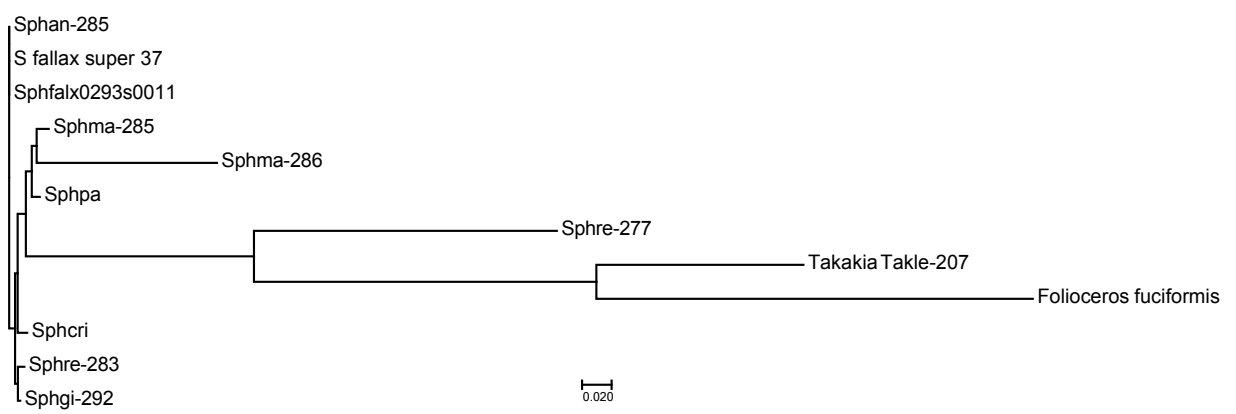

Figure 2 Analysis of TAS3 loci in genus Sphagnum. (A) Multiple sequence alignment of available nucleotide sequences of TAS3-like loci from mosses of genus Sphagnum along with TAS3 loci of Takakia lepidozioides and Folioceros fuciformis. Alignment was generated at MAFFT6 program. The miR390 target sites are in yellow, and putative tasiARF-a2 site is in green; tasiAP2 site is in blue. (B) The minimal evolution phylogenetic tree based on analysis of the aligned TAS3 genes from mosses of genus Sphagnum. This tree was generated according MAFFT6 program. For full plant names and accession numbers see Table 1.

Full-size DOI: $10.7717 /$ peerj.4636/fig-2 


\begin{tabular}{|c|c|c|c|c|}
\hline Plant species & Locus name & Subgenus & Length & Sequence source \\
\hline Sphagnum angustifolium & Sphan-285 & Cuspidata & 285 nts & MF682529 \\
\hline S. girgensohnii & Sphgi-292 & Acutifolia & $292 \mathrm{nts}$ & MF682530 \\
\hline S. fallax & contig super_37 & Cuspidata & $285 \mathrm{nts}$ & SRX2120232 \\
\hline S. fallax & Sphfalx0293s0011 & Cuspidata & $277 \mathrm{nts}$ & Sphfalx0293s0011 \\
\hline S. recurvum & Sphre-283 & Cuspidata & $283 \mathrm{nts}$ & SRX1513231 \\
\hline S. recurvum & Sphre-277 & Cuspidata & $277 \mathrm{nts}$ & SRX1513231 \\
\hline S. magellanicum & Sphma-285 & Sphagnum & $285 \mathrm{nts}$ & SRX2330962 \\
\hline S. magellanicum & Sphma-286 & Sphagnum & $286 \mathrm{nts}$ & SRX2330962 \\
\hline S. palustre & Sphpa & Sphagnum & partial & SRX1516347 \\
\hline S. cribrosum & Sphcri & Subsecunda & $291 \mathrm{nts}$ & ERX443237 \\
\hline S. lescurii & Sphle & Subsecunda & partial & ERX337183 \\
\hline Takakia lepidozioides & Takle-207 & Not applicable & $207 \mathrm{nts}$ & $\begin{array}{l}\text { ERX2100030 } \\
\text { SKQD-2076588 }\end{array}$ \\
\hline
\end{tabular}

was revealed in a longer assembly which was found recently upon search of $1 \mathrm{KP}$ database (Xia, Xu \& Meyers, 2017).

Since Takakiopsida and Sphagnopsida are most basal sister lines to all other Bryophyta (Shaw et al., 2010; Shaw, Szövényi \& Shaw, 2011; Rosato et al., 2016; Puttick et al., 2018), it was very interesting to compare the structural organization of Takakiopsida and Sphagnopsida TAS3 loci with other classes of Bryophyta. Our previous detailed analysis of approximately 40 TAS3 loci in Bryophyta (Krasnikova et al., 2011; Krasnikova et al., 2013) showed that the general structure of moss TAS3 is similar in all taxa and fits the structural organization of Physcomitrella patens genes, comprising dual miR390 target sites on the $5^{\prime}$ and $3^{\prime}$ borders and internal monomeric tasiAP2 sequence followed by tasiARF sequence positioned in 20-30 bases. We revealed that phylogenetic tree of TAS3-like loci in Bryophyta showed clear subdivision of their sequences into two main clades (see Fig 5 in Krasnikova et al., 2013). The first group was formed by a cluster of sequences close to P. patens TAS3 species PpTAS3a, PpTAS3d, and PpTAS3f, and the second one-by those close to PpTAS3b, PpTAS3c, and PpTAS3e. The recent paper on the structure of TAS3 loci in lower land plants (Xia, Xu \& Meyers, 2017) has shown the structure-functional basis for this phylogenetic subdivision. TAS3 species of the first group (PpTAS3a/PpTAS3d/PpTAS3f cluster) were shown to form class III of TAS3-like loci and contain, in addition to the previously reported tasiAP2 and tasiARF-a2 sequences, newly discovered tasiARF-a3 sequence positioned 5' according to tasiAP2 (Fig. 3). Among TAS3 species of basal Bryophyta, Andreaea rupestris locus 13-Aru (Krasnikova et al., 2013) belongs to class III (Fig. 3).

BLAST comparison of T. lepidozioides TAS3 with known Bryopsida loci showed that Takle-207 belongs to class II of TAS3 with typical positioning of tasiAP2 and tasiARF-a2 sequences (Fig. 2 and Fig. S1). On the other hand, none of Sphagnopsida TAS3-like sequences (Table 1) showed conventional internal structural organization 
Folioceros

32-Oxy

50-Br

11-Pyp

28-Bha

72-Pps

9-Bar

31-Erh

29-Bha

9-Tau

80-Tpe

TAS3a

24-Opu

TAS3d

35-Erh

13-Aru

Folioceros

32-Oxy

$50-\mathrm{Br}$

11-Рyp

28-Bha

72-Pps

9-Bar

31-Erh

29-Bha

9-Tau

80-Tpe

TAS3a

24-Opu

TAS3d

$35-\operatorname{Erh}$

13-Aru
CTTGTTAACGGGGTGTTAAGCACCAACGGACGCCCTGGCAGCCTCAGACGCCACCCACGGCTCCGTAGGGTGTGATGAGTGCTTTACCTAGCG CTTGTTAACGGGGTGTTAAGCACCAACGGGCGCCCCGGTGGCCTCAGATGTCCGCCATGGCTCCGTAGGGTGTGATGAGTGCTTTACCCAGCA CTTGTTAACGGGGTGTTAAGCACCAACGGGCGCCCCGGTGGCCTCAGATGTCTGTTATGGCTCCGTAGGGTGTGATGAGTGCTTTACCCAACA CTTGTTAACGGGGTGTTAAGCACCAACGGACGTCCCGGCAGCCTCAGACGTCACCCATGGCTCCGTAGGGTGTGATGAGTGCTTTACCCGGCA CTTGTTAGCGGGGTGTTAAGCACTAGCGTGCGGCACTGCGCCTTAAGACGTCAGCTATGGCTCCGTAGGGTGTGATGAGTGCTTTACCCGGCA CGTGTTAGCGGGGTGTTGAGCACTGAAGTGCGGACCTCCAGCTGCAGACGTTAGCTATGGCTCCGTAGGGTGTGATGAGTGCTTTACCAGGCG CTTGTTAGCGGGGTGTTAAGCACTTCTGTGCGAACCTCCATCTCAAGACGCCAGCTACGGCTTCGTAGGGTGTGATGAGTGCTTTAAATGGCG CTTGTTAGCGGGGTGTTAAGCACTTGAGTGCAACACTCCGGCCTAAGACGTCAGCTATGGCTTCGTAGGGTGTGGTGAGTGCTTTACCCAGCA CTTGTTAGCGGGGTGTTAAGCACTTGAGTACGACACTCGGGCCCTTGACCTCCGCTATGGCTTCGTAGGGTGTGATGAGTGCTTTACCCGGCG CTTGTTAGCGGGGTGTTAAGCACTTGAGTACGACACTCGGGCCCTTGACCTCCGCTATGGCTTCGTAGGGTGTGATGAGTGCTTTACCCGGCG CTTGTAGGCGGGGTGTTAAGCACTCGCGTGCGGCTCTCCGTGGTAAGACGTCAGCTATGGCTCCGTAGGGTGTGATGAGTGCTTTACCTGGCG CTTGTTAGCGGGGTGTTAAGCATTTGAATGCAACACTCCTACGCAAGACCCTAGCTATGGCTCCATAGGGTGTGATGAGTGCTTCATCCGGTG CATGTTAGCGGGGTGTTAAGCACACGAGCCCGGCAGAGCGGCGCCAACCGTGAGGCATGGCCCTGTAGGGTGTGATGAGTGCTTTACACGGCG CTTGTAAGCGGGGTGTTATGCACTTGCGCTTGACGCTCCGATCTAAGACGTCAGCTGTAGCTCCATGGGGTGTGATGACTGCTTAGCCCGGCA CTTGTAAGCGGGGTGTTACGCACAAGGGTGTGCCACTCCCGCCTAAGACGTCAGCTATGGCTCTGTAGGGTGTGATGAGTGCTTTACCGAGCG CTTGTAGGCAAGGTGTTAAGCACTTTAGTGCGAGACCCTGCCACAAGACGCTAGCTACAGCTCCCTAGGGTGTGATGAGTGCTTTAGCTGGCA

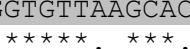

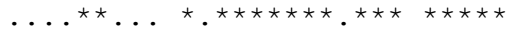

$\cdots$

CTCAGCCCCTGGCGAGC

CTCATCCTCTACGCAGCC

CTCATCCTCTACGCAGCC

CTCATCCTCTACGCAGCCCACCTACCCTTGT

CTCATCCTCTACCCAGC

СTCATCCTCTACCCAGCCCACCTACCCTTGT

CTCATCGACCGCCCTGC

CTCATCCACTGCCCAGCCCACCTACCCTTGT

CTCATCCACTGCCCAGC

СТСААСААСTTCCCAGCCCACCTACСCTTGG

CTCTTCTACTGCCTTGC

CTCGTCCTCTGCCCAGCCCACCTACCCTTGT

CACACTCTCTGCCCGGC

СTCATCCACCGCCTTGAACACCTACCCTAGC

CTCATCCACTACCCA

$\star \star$

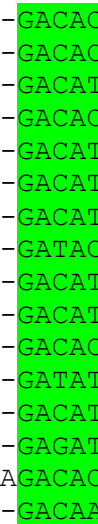

GGGCCTGGCAGATCCCTGCACGGCCCCTGTCGG-TTACGTATCACTCCTGAGCTA GGGCGAAGCGCTTATCAGCGCAGCCCGTGTCAA-TTGTATATCACTCCTGAGCTA GGGCGCAGTGCTTATCGGCGCAGCCCATGTCAA-TTGTATATCACTCCTGAGCTA GGGCGCAGCGCTTCCCGGTACAGCCCGTGTCAA-TTGTATATCACTCCTGAGCTA GGGTCGCGCTCTTCCGGGCGCAGCCCATGTCAA-TTGTCTATCACTCCTGAGCTA GGGCCGCTCCCTTCCCGGCGCGGCCCTTGTCAA-TTGTCTATCACTCCTGAGCTA GGGCCGCTCCCCTTCCGGTTCGGCCCTTGTCAA-TTGTCTATCACTCCTGAGCTA GAGCCTCGCAGATTCCTGCGTGGCCCGTGTCGG-TTGTATATCACTCCTGAGCTA GGGCACCGCAGATCCCTGCGCTGCCCTTGTCGG-TTGTATATCACTCCTGAGCTA GGGCACCGCAGATCCCTGCGCTGTCCTTGTCGG-TTGTATATCACTCCTGAGCTA GGGCCAAGTGGATTTCCACTCGGCCTGTGTCGG-TTGTCTATCACTCCTGAGCTA GGGCCGCGCGTGTCTGCGTGTCTCCTGTATCGG-TTGTATATCACTCCTGAGCTA GGGCACCGCCCTTAGGGGCGCCGTCCGTGTCGA-TTGTCTATCACTCCTGAGCTA GGGTAGCGCTGATTTGCGCGACTTCCATGTCGG-TTTTATATCACTCCTGAGCTA GGCCCTCGCAGTTTCTTGCGTGGCCCGTGGTCA-TTGTCTATCACTCCTGAGCTA AGGCTGTGC-TACCTCTGCGCAGTCCCTGTCGGTTTGTATATCACTCCTGAGCTA

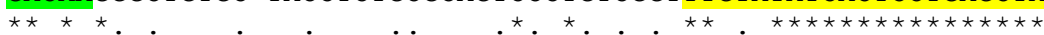

Figure 3 Multiple sequence alignment of selected available nucleotide sequences of class III TAS3-like loci from Bryopsida mosses along with TAS3 locus of Folioceros fuciformis. Alignment was generated at MAFFT6 program. The miR390 target sites are in yellow; putative tasiARF-a2 site is in green; tasiAP2 is in blue, and tasiARF-a3 is shaded. Note that all sequences are cut in the $5^{\prime}$-terminal TAS3 area to exclude non-aligned regions.

Full-size DOI: 10.7717/peerj.4636/fig-3

of the most moss TAS3 species. The only recognizable conserved site, except miR390targeting regions, was identified as tasiARF-a2 sequence, which was found to be conserved between two very distant TAS3 loci in S. fallax and S. recurvum (Fig. 2). The mentioned above tasiARF sequences, tasiARF-a2 and tasiARF-a3, showed no sequence similarity suggesting their independent origins. These tasiRNAs were found to be formed from different strands of the TAS3 dsRNA intermediate and target different regions of ARF genes (Xia, Xu\& Meyers, 2017). Inhibition of production of both tasiARF RNAs in $P$. patens resulted in obvious developmental defects exhibited, in particular, as alterations in gametophore initiation, protonemal branch determinacy and caulonemal differentiation (Plavskin et al., 2016).

Comparison of nucleotide sequences between TAS3 species of several moss classes revealed in many plants obvious similarity of nucleotide sequence blocks including tasiAP2 site and immediate upstream 21 bp block occurring in the same 21-bp-phase (Fig. 4A). We hypothesized that this sequence block may correspond to novel previously unrecognized 
(A)

\section{Andreaeal Andreaea2 Tetraphis 1 Tetraphis2 Tetraphis 3 Plagiomnium Leucobryum Racomitrium Philonotis Dicranum Encalypta Ceratodon Niphotrichum Funaria Schwetschkeop Aulacomnium Syntrichia Diphyscium1 Diphyscium2 Pohlia Bryum PHYSCO TAS3A}

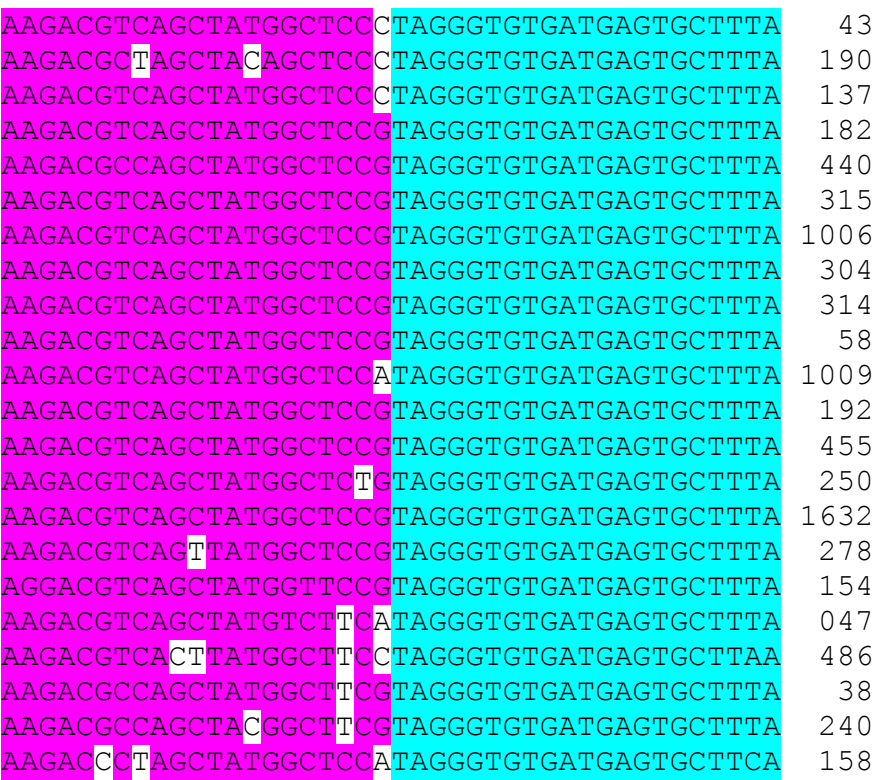

(B)

$3^{\prime}$-UUCUGCaGUCGAUaCCGAGGc caagacagttacggatcatctggacgtaccggaggtggtctagggcaagatgatagctacggctcat $\begin{array}{lllllllllllllllllllllllllllllllll}Q & D & S & Y & G & S & S & G & R & T & G & G & G & L & G & Q & D & D & S & Y & G & S & - & 28\end{array}$ 3' -UUCUGCaGUCGAUACCGAGGC

tcatctggtcgcactggaggtggcctcggccaagacgacagctatggttcctctggccgc $\begin{array}{llllllllllllllllllllll}S & S & G & R & T & G & G & G & L & G & Q & D & D & S & Y & G & S & S & G & R & - & 56\end{array}$ 3'-UUCUGCaGUCGAUaCCGAGGc

ggtggcggcctcggtaaagatgatagctacggctcctcaggacgtaccgggggaaataca $\begin{array}{llllllllllllllllllllllllll} & G & G & G & L & G & K & D & D & S & Y & G & S & S & G & R & T & G & G & N & T & - & 96\end{array}$ 3' -UUCUGCaGuCGAUACCgAGGc

cttggacaagatgattcgtatggatcctctggacaaactggcggtaacagcggatacggc

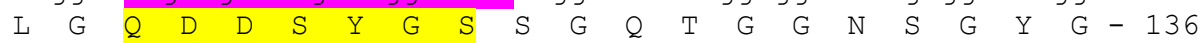

Figure 4 Novel potential ta-siRNA in Bryopsida plants. (A) Multiple sequence alignments of nucleotide sequence blocks including tasiAP2 site and preceding 21 bp site of putative ta-siRNA of Andreaea rupestris TAS3-like locus WOGB_2010369 with the corresponding transcript sequences of moss TAS3 loci. BLASTN was used at $1 \mathrm{KP}$ blast site. For the complete TAS3 transcript sequences see Xia, Xu \& Meyers, 2017. The putative tasiAP2 site is in blue, and preceding putative ta-siRNA site is in violet. Andreaeal - Andreaea rupestris WOGB_2010369; Andreaea2 - Andreaea_rupestris WOGB_2002765; Tetraphis1 - Tetraphis_pellucida HVBQ_2019753; Tetraphis2 - Tetraphis_pellucida HVBQ_2011866; Tetraphis3 Tetraphis_pellucida HVBQ_2005644; Plagiomnium - Plagiomnium_insigne BGXB_2010105; Leucobryum - Leucobryum_glaucum RGKI_2062694; Racomitrium - Racomitrium_varium RDOO_2117129; Philonotis - Philonotis fontana ORKS_2058791; Dicranum - Dicranum_scoparium NGTD_2078536; Encalypta -Encalypta streptocarpa KEFD_2058811;(continued on next page...)

Full-size DOI: 10.7717/peerj.4636/fig-4 
Figure 4 (...continued)

Ceratodon - Ceratodon_purpureus FFPD_2044193; Niphotrichum - Niphotrichum_elongatum ABCD_2000143; Funaria - Funaria sp. XWHK_2042016; Schwetschkeop-Schwetschkeopsis fabronia IGUH_2166854; Aulacomnium - Aulacomnium heterostichum WNGH_2088134; Syntrichia Syntrichia_princeps GRKU_2074985; Diphyscium1 - Diphyscium foliosum AWOI_2069791; Diphyscium2 - Diphyscium foliosum AWOI_2006305; Pohlia - Pohlia nutans GACA01023180; Bryum argenteum Unigene33538 GCZP01053768; PHYSCO TAS3A -Physcomitrella patens TAS3a BK005825. (B) The example target transcript sequence (lower case letters) from Leucobryum albidum (VMXJ_2127900) is presented alongside with the predicted novel ta-siRNA shown in blue and above the transcript. Lower case letters in ta-siRNA indicate non-Watson-Crick pairing positions. Complementary mRNA sequences are in violet; conserved amino acid sequence signatures are in yellow. Numbers indicate codon positions of the target gene.

ta-siRNA in many moss species. This putative siRNA in its single-stranded form really presents in $P$. patens transcriptome as minus-sense 21-nucleotide ta-siRNA (see NCBI SRA accessions SRX903096-SRX903105) like tasiARF RNA (Arif et al., 2012). Thus, we speculated that novel hypothetical ta-siRNA might be produced from TAS3, and its minus-strand is complementary to uncharacterized well-conserved, protein-coding moss mRNAs which have homologs also in conifers and angiosperms (Fig. 4B; Fig. S2).

\section{TAS3 loci in Anthocerotophyta}

Taking into account the finding of TAS3-like loci in classes Sphagnopsida and Takakiopsida and previously published data (Krasnikova et al., 2013; Xia, Xu\& Meyers, 2017), one can conclude that the only remaining blind-spot in land plants with respect to TAS3 is represented by phylum Anthocerotophyta. Relationships between liverworts, mosses and hornworts are still obscure. Moreover, the question remains which bryophyte phylum is a sister line to all other land plants (Qiu, 2008; Shaw, Szövényi \& Shaw, 2011; Harrison, 2017; Puttick et al., 2018). Recent molecular phylogenetic analysis, in which three bryophyte lineages were resolved, revealed that a clade with mosses and liverworts could form a sister group to the tracheophytes, whereas the hornworts is sister line to all other land plants (Wickett et al., 2014). However, analyses of the plastid genome sequences suggested another branching order of the phylogenetic tree, with hornworts rather than moss/liverwort clade being a sister group to tracheophytes (Lewis, Mishler \& Vilgalys, 1997; Samigullin et al., 2002; Ruhfel et al., 2014; Lemieux, Otis \& Turmel, 2016). Moreover, some very recent nuclear gene comparisons also suggested that hornworts could be a sister clade to tracheophytes, and liverworts plus mosses might be closer to a common ancestor of land plants (Rosato et al., 2016; Bowman et al., 2017). However, this ancestor could have more tracheophyte-like characteristics than Setaphyta (liverworts/mosses) due to secondary simplification in liverworts (Puttick et al., 2018).

Analysis of the SRA database of Anthocerotophyta revealed a putative TAS3-like sequence in Folioceros fuciformis (family Anthocerotaceae). Unexpectedly, the discovered TAS3-like sequence (Folfu) was found to be 244 nucleotides in length and obviously similar to Bryophyta class III TAS3 species (Fig. 3, Fig. S3 and Table 2). The identity of Folfu to some moss TAS3 sequences exceeds $80 \%$ being therefore even higher than between some related Bryopsida species (Fig. 3). Thus these data clearly indicate a close relation of TAS3 in Anthocerotophyta to Bryophyta TAS3 (excepting Sphagnopsida). 
Table 2 List of the putative TAS3 loci in Anthocerotophyta and Marchantiophyta.

\begin{tabular}{|c|c|c|c|c|}
\hline Plant species & Class/subclass & Order & Length & Sequence source \\
\hline Folioceros fuciformis & Anthocerotopsida/Anthocerotidae & Anthocerotales & $244 \mathrm{nts}$ & SRS2162762 \\
\hline $\begin{array}{l}\text { Marchantia polymorpha } \\
\text { 1-Mpo }\end{array}$ & Marchantiopsida/Marchantiidae & Marchantiales & $256 \mathrm{nts}$ & KC812742 \\
\hline Marchantia emarginata & Marchantiopsida/Marchantiidae & Marchantiales & $262 \mathrm{nts}$ & SRX1952816 \\
\hline Conocephalum japonicum & Marchantiopsida/Marchantiidae & Marchantiales & $252 \mathrm{nts}$ & SRX1952810 \\
\hline Ricciocarpos natans & Marchantiopsida/Marchantiidae & Marchantiales & $235 \mathrm{nts}$ & ERX337127 \\
\hline Dumortiera hirsuta & Marchantiopsida/Marchantiidae & Marchantiales & $243 \mathrm{nts}$ & SRX1126014 \\
\hline Plagiochasma appendiculatum & Marchantiopsida/Marchantiidae & Marchantiales & 247 nts & SRX1741567 \\
\hline Conocephalum conicum & Marchantiopsida/Marchantiidae & Marchantiales & $248 \mathrm{nts}$ & ILBQ_2006554 \\
\hline Lunularia cruciata & Marchantiopsida/Marchantiidae & Lunulariales & 220 nts & TXVB_2071521 $1^{\mathrm{a}}$ \\
\hline Marchantia paleaceae & Marchantiopsida/Marchantiidae & Marchantiales & $257 \mathrm{nts}$ & HMHL_2051051 \\
\hline Metzgeria crassipilis & Jungermanniopsida/Metzgeriidae & Metzgeriales & $226 \mathrm{nts}$ & ERX337128 \\
\hline Pellia endiviifolia & Jungermanniopsida/Pelliidae & Pelliales & $192 \mathrm{nts}$ & SRX726500 \\
\hline
\end{tabular}

Notes.

${ }^{a} 1 \mathrm{KP}$ accession (Xia, Xu \& Meyers, 2017).

\section{TAS3 loci in Marchantiophyta}

Some of the recent molecular phylogenetic reconstructions suggested that Marchantiophyta species could represent a sister clade to all other land plants (see above). Therefore, finding and comparative analyses of TAS3 loci in this taxon represented a significant interest for understanding early events in TAS3 evolution. In contrast to class Marchantiopsida, where putative TAS3 and pre-miR390 loci were previously identified (Krasnikova et al., 2013; Lin et al., 2016; Tsuzuki et al., 2016), for class Jungermanniopsida only potential pre-miR390 loci were found in Pellia endiviifolia and Harpanthus flotovianus (Krasnikova et al., 2013; Alaba et al., 2015). Assuming that miR390 was found to be among eight most conserved miRNA species in land plants (Xia et al., 2013; You et al., 2017; Liu et al., 2018), Jungermanniopsida could be expected to encode TAS3 loci.

To detect new potential TAS3 loci, we performed BLAST analysis of the SRA database for species of class Jungermanniopsida using Marchantia polymorpha TAS3 sequence (1-Mpo) as a query. Using this approach we revealed a set of reads and assembled a single TAS3-like locus (Pelen-192) for Pellia endiviifolia (192 nt size). In addition, TAS3 locus of 226 nucleotides in length was found in Metzgeria crassipilis (Metcr-226) (Fig. 5, Table 2, Fig. S3). The latter locus was also recently revealed in a search of $1 \mathrm{KP}$ database (Xia, Xu \& Meyers, 2017).

TAS3 1-Mpo sequence was further used for BLAST analysis of other Marchantiopsida sequences available at the NCBI SRA database. As a result, we retrieved sequence reads and assembled five full-length TAS3-like sequences in Plagiochasma appendiculatum (Plaap247), Dumortiera hirsuta (Dumhi-243), Marchantia emarginata (Marem-262), Ricciocarpos natans (Ricna-235) and Conocephalum japonicum (Conja-252) (Fig. 5, Table 2, Fig. S3). Recent bioinformatics analysis of $1 \mathrm{KP}$ database revealed three additional full-length TAS3like sequences in Conocephalum conicum, Lunularia cruciata and Marchantia paleaceae 
(A)

Takakia

Folioceros

1-Mpo

Marchantia

Conocephal. Jap

Conocephal.

Dumortiera

Rlagiochasma

Ricciocarpos
Lunularia

Lunularia

Pellia

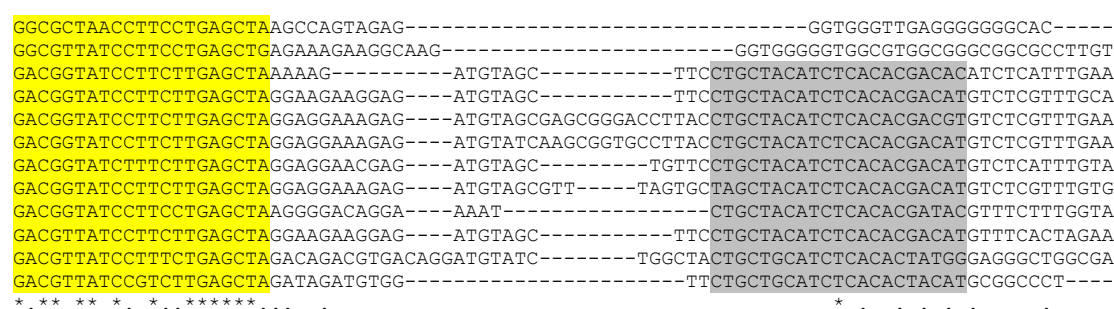

Takakia

Folioceros

1-Mpo

Marchantia

Conocephalum

Conocephalum

Dumortiera

Plagiochasma

Ricciocarpos

Lunularia

Metzgeri

Pellia

(B)

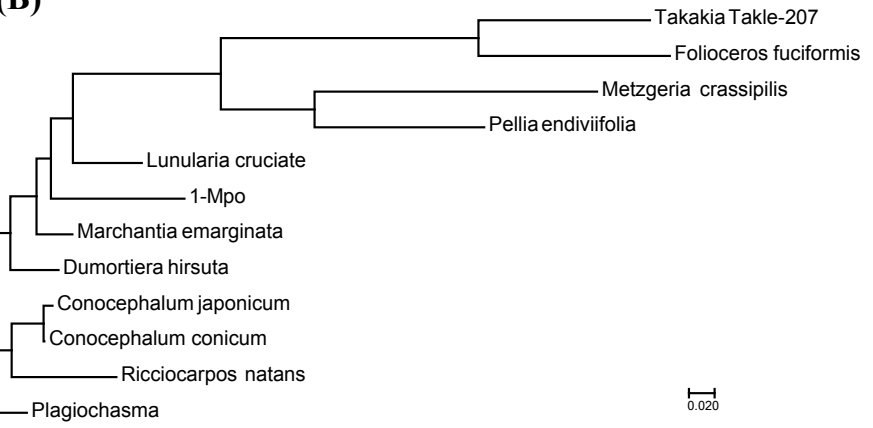

Figure 5 Analysis of TAS3 loci in Marchantiophyta plants. (A) Multiple sequence alignment of available nucleotide sequences of TAS3-like loci from Marchantiophyta plants along with TAS3 loci of Takakia lepidozioides and Folioceros fuciformis. Alignment was generated at MAFFT6 program. The miR390 target sites are in yellow, and putative tasiARF-a2 site is in green; tasiAP2 site is in blue. (B) The minimal evolution phylogenetic tree based on analysis of the aligned TAS3 genes from Marchantiophyta plants. This tree was generated according MAFFT6 program. For full plant names and accession numbers see Table 2. 
(Xia, Xu \& Meyers, 2017) (Table 2). Thus, totally 11 TAS3-like loci have been found in Marchantiophyta.

\section{TAS6 loci in Bryophyta}

Previous studies of $P$. patens revealed three novel non-coding PHAS loci (TAS6) which were located in rather close genomic proximity to PpTAS3 loci (PpTAS3a, PpTAS3d, and PpTAS3f) and expressed as common RNA precursors with these TAS3 species (Cho, Coruh \& Axtell, 2012; Arif et al., 2012; Arif, Frank \& Khraiwesh, 2013). Moreover, miR529 and miR156 were suggested to influence accumulation of ta-siRNAs specific not only for TAS6, but also for PpTAS3a (Cho, Coruh \& Axtell, 2012). We have found that localization of TAS6 loci close to TAS3 genes in common transcripts was not unique for P. patens (subclass Funariidae), since these loci were also found to be encoded by three other mosses of subclasses Bryidae and Dicranidae (Krasnikova et al., 2013).

For further search of the combined TAS6/TAS3 loci, we performed bioinformatics analysis of 1KP database. Although nucleotide sequences of miR156 and related miR529, as well as their recognition sites in RNA transcripts, are highly conserved among land plants (Morea et al., 2016; Axtell \& Meyers, 2018), the internal sequences between dual miR156/miR529 recognition sites show little or no similarity even between different TAS6 loci of $P$. patens (Arif et al., 2012). So we used, as queries for BLAST search, the individual full-length TAS6/TAS3 loci including most characterized locus encoding PpTAS3a (Fig. 6), as well as those for PpTAS3d and PpTAS3f. First, it was found that in addition to four previously found Bryopsida species, encoding TAS6/TAS3 loci, these loci could be revealed in basal subclasses Timmiidae (Timmia austriaca) and Diphysciidae (Diphyscium foliosum) (Shaw, Szövényi \& Shaw, 2011; Table 3, Fig. S4). List of TAS6/TAS3 loci in other moss subclasses was also significantly extended: we found 18 new loci in Bryidae, seven loci in Dicranidae and four loci in Funariidae (Table 3, Fig. S4). These novel loci showed recognizable but varying sequence similarities to the PpTAS3a-containing locus (Fig. 6). Second, most importantly, putative TAS6/TAS3 loci were revealed in four basal classes of Bryophyta, namely, Tetraphidiopsida, Polytrichopsida, Andreaeopsida and Takakiopsida (Table 3, Fig. S4). These novel loci had a similar organization to Bryopsida TAS6/TAS3 species (Fig. 6). However, no TAS6-specific sequence signatures were found in the vicinity of genomic $S$. fallax and $M$. polymorpha TAS3 loci upon analysis of the corresponding Phytozome genome contigs.

\section{Phylogeny of SGS3 as a characteristic molecular component of TAS3 pathway}

It was shown that some species of green algae could encode ancient types of dicer-like proteins, RDRs, and AGOs. On the other hand, no encoded SGS3 proteins were revealed for these algae (Zheng et al., 2015). Since SGS3 was found to be essential for production of tasiARF RNAs in moss P. patens (Plavskin et al., 2016), we performed sequence analyses to identify possible SGS3 genes in charophytes. For identification of SGS3 protein orthologs among land nonvascular plants and charophytes, we used as a query the most conserved region of $P$. patens SGS3 including short zinc binding zf-XS domain and RNA recognition 
2058811 Encalypta streptocarpa (accession KEFD_2058811)

\begin{tabular}{|c|c|c|c|}
\hline Query & 1 & $\begin{array}{l}\text { ACTCTTCATATGTGCTCTCTCTCTTCA---CTGTCAAGACCTCGCTTTCGGTCAGCTGCA } \\
|||||||||||||||||||||||| \mid\end{array}$ & 57 \\
\hline Sbjet & 212 & ACTCTTCACACGCGCTCTCTCTCCTCAGTGCTGTCATGATCGCGCTTTCGGGCTGCTGCA & 271 \\
\hline Query & 58 & TGTCAGACTGCTTGAAGGCCGAGG-AGAACA-TCTC---T----AACGCGGTCGCTTCTT & 108 \\
\hline Sbjet & 272 & 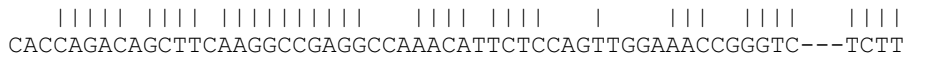 & 328 \\
\hline Query & 109 & $\begin{array}{l}\text { GTACCCATCAAAAGCTTCA--TTA-AGCTGCTG-TCGA----CAGGGGCACTGCACCCT- } \\
|||||||||||||||| \quad||||||||||||||||||||||\end{array}$ & 159 \\
\hline Sbjet & 329 & GTACCCGAC-CAAGCTTCACCGTATTGTCGGTGAACGATAATCAGCGACACCGCGCTGTC & 387 \\
\hline Query & 160 & 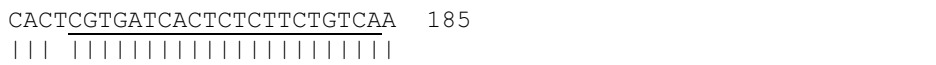 & \\
\hline Sbjet & 388 & CAC-CGTGATCACTCTCTTCTGTCAA & \\
\hline Query & 615 & 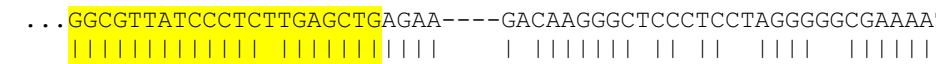 & \\
\hline Sbjet & 855 & ....GGCGTTATCCCTCCTGAGCTGAGAAAGTTGGCAAGGGCCCCATC--AGGG--CGAAAA & $\mathrm{TA}$ \\
\hline Query & 671 & $\begin{array}{l}\text { GGTGAGCTGGGGTCACCTTGTTAGCGGGGTGTTAAGCATTTGAATGCAACACTCCTACGC } \\
|||||||||||||||||||||||||||||||||||||||||||||||||||||||| \mid\end{array}$ & 730 \\
\hline Sbjet & 911 & GGTGCGCCGACTTC---TTGTTAGCGGGGTGTTAAGCACTTGAGTGCGACACTCCGACCT & 967 \\
\hline Query & 731 & $\begin{array}{l}\text { AAGACCCTAGCTATGGCTCCATAGGGTGTGATGAGTGCTTCATCCGGTGCTCTTCTACTG } \\
|||||||||||||||||||||||||||||||||||||||||||||||||||||||||||| l||\end{array}$ & 790 \\
\hline Sbjet & 968 & AAGACGTCAGCTATGGCTCCATAGGGTGTGATGAGTGCTTTATCCGACACTCATCGACCG & 102 \\
\hline Query & 791 & $\begin{array}{l}\text { CCTTGCCCACCTACCCTTGTGATATGGGCCGCGC-G-TGTCTGCGTGTCTCCTGTATCGG } \\
|||||||||||||||||||||||||||||||||||||||||||||||||||||||||| l \mid\end{array}$ & 848 \\
\hline Sbjet & 1028 & CСCTGCCCACCTACCCTTGTGATACGGGCTTCGCAGATTCCTGCGTGGC-CC-GTGTCGG & 108 \\
\hline Query & 849 & 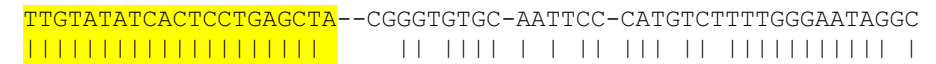 & 904 \\
\hline Sbjet & 1086 & TTGTATATCACTCCTGAGCTTATAAGGAGTGCGAGTGCCGCATCTCCTTTGGGAATAGCC & 114 \\
\hline
\end{tabular}

\section{Philonotis fontana (accession ORKS_2058791)}

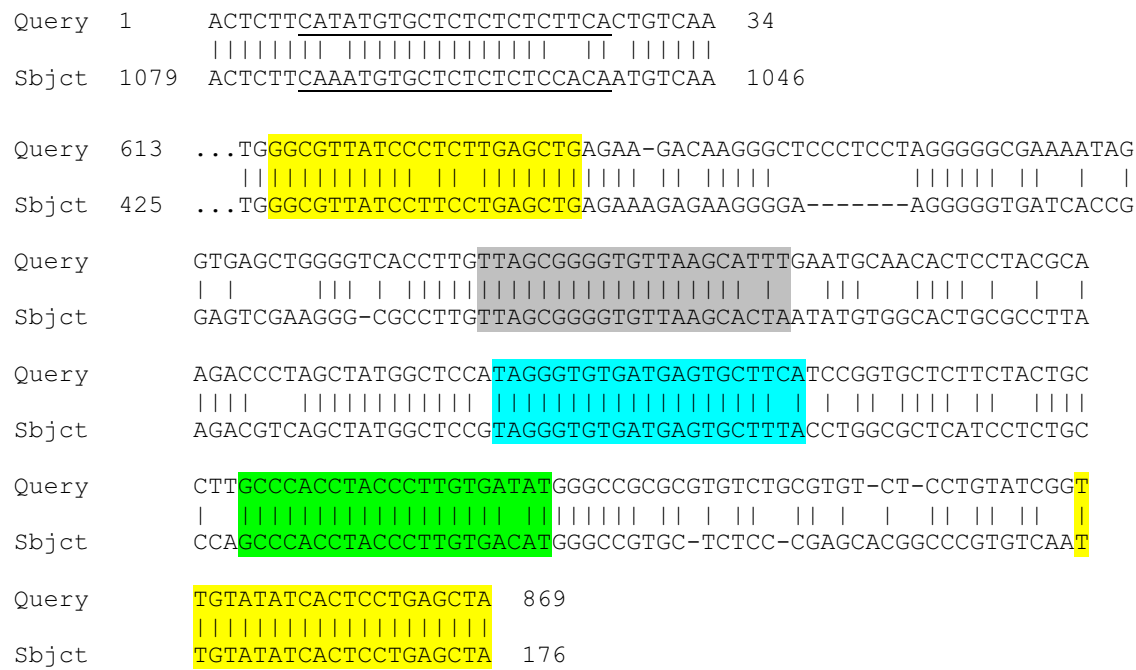

Figure 6 Pairwise sequence comparisons of selected nucleotide sequences of TAS6/TAS3-like loci from mosses with TAS6/TAS3 of Physcomitrella patens precursor RNA (accession JN674513). BLASTN was used at $1 \mathrm{KP}$ blast site. The miR390 target sites are in yellow; putative miR156/miR529 sites are underlined; tasiAP2 is in blue; putative tasiARF-a2 site is in green; tasiARF-a3 is shaded.

Full-size DOI: 10.7717/peerj.4636/fig-6 


\section{Hedwigia ciliata (accession YWNF_2050742)}

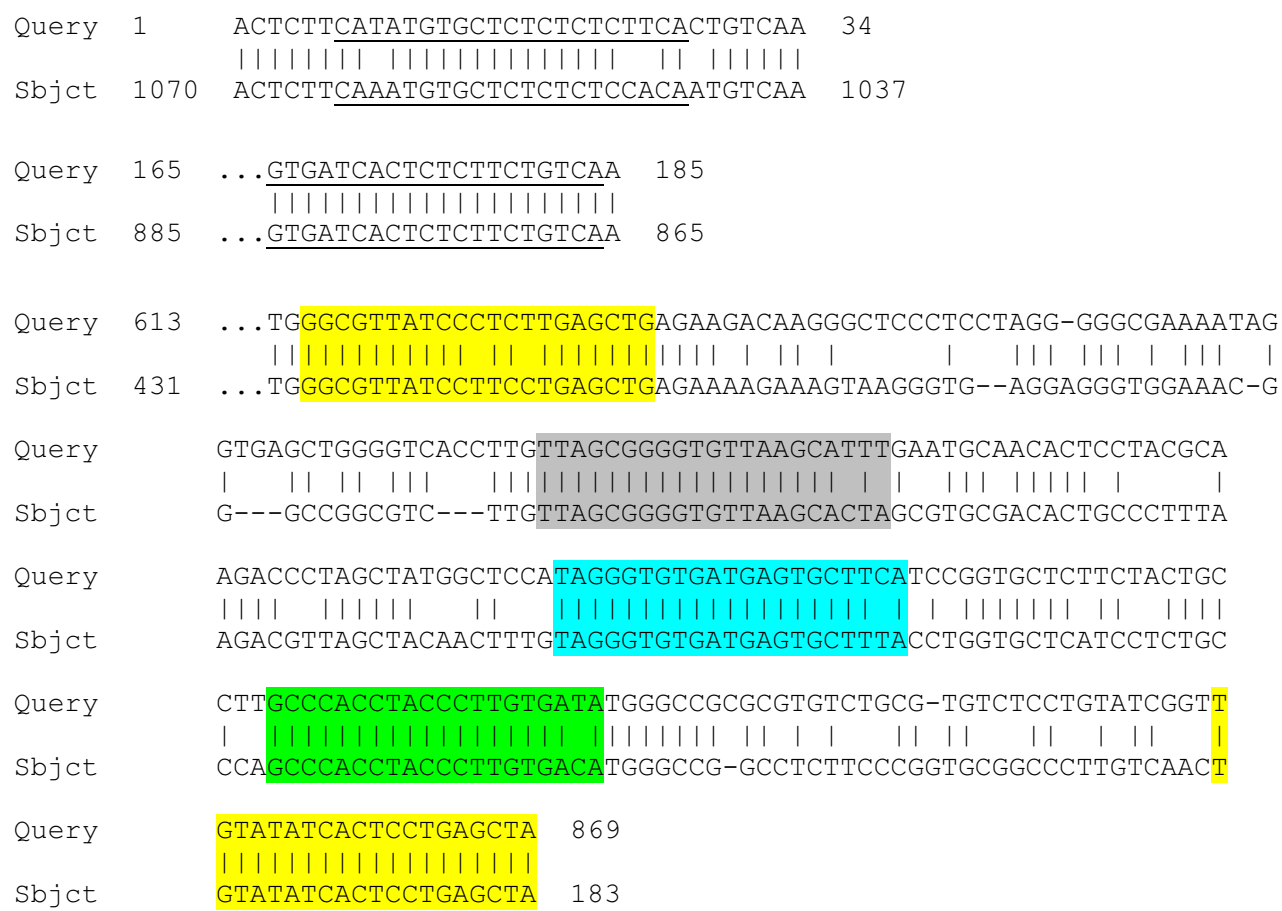

2069791 Diphyscium foliosum (accession AWOI_2069791)

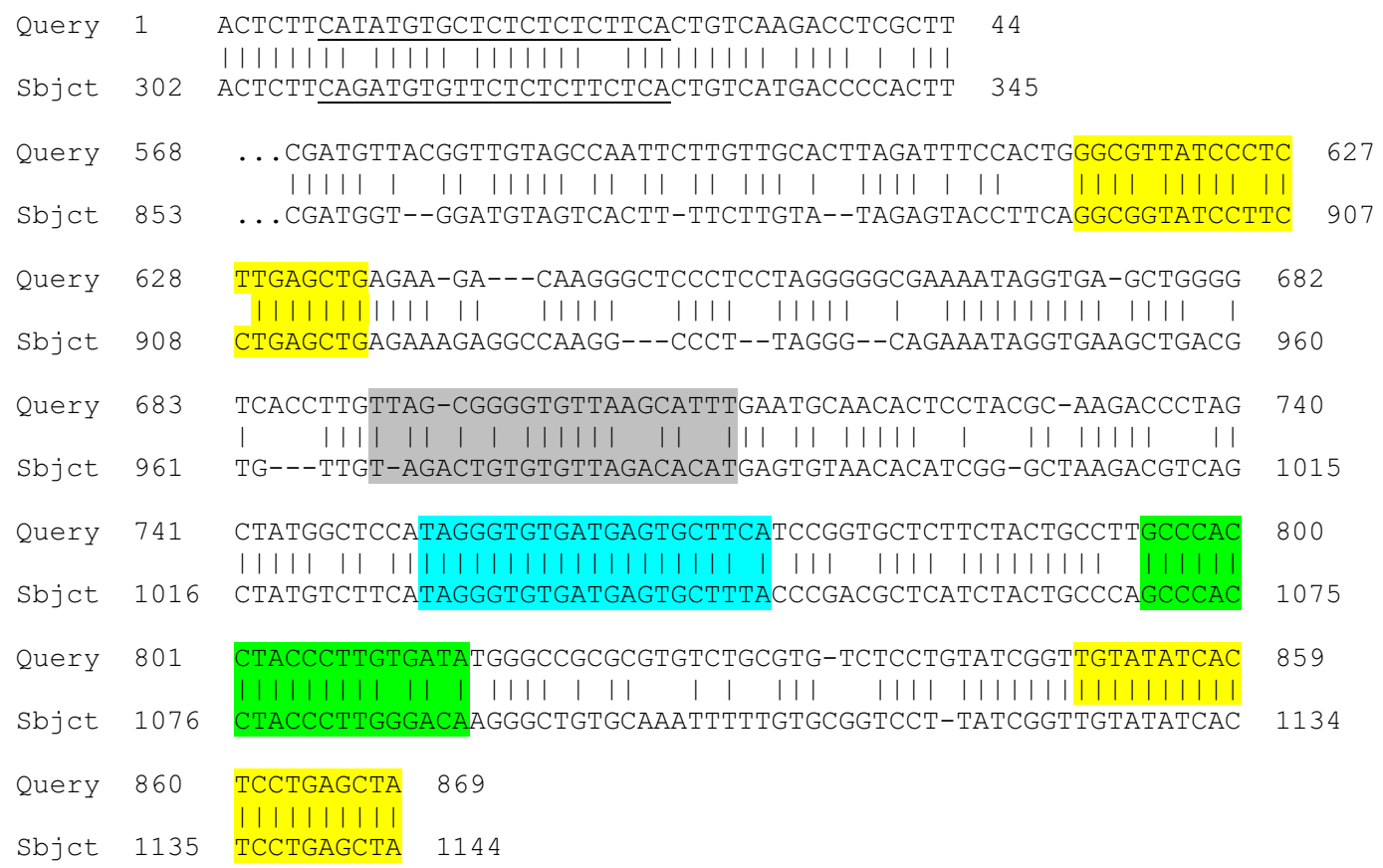

Figure 6 (...continued) 
Table 3 List of the putative TAS6/TAS3 loci of Bryophyta in transcribed sequences found in $1 \mathrm{KP}$ database.

\begin{tabular}{|c|c|c|c|c|}
\hline Plant species & Class/subclass & Order & Length ${ }^{\mathrm{a}}$ and type & Sequence source \\
\hline Timmia austriaca & Bryopsida/Timmiidae & Timmiales & TAS6/TAS3 (874 nts) & $\begin{array}{l}\text { ZQRI-2061439 } \\
\text { ZQRI-2063082 }\end{array}$ \\
\hline Thuidium delicatulum & Bryopsida/Bryidae & Hypnales & TAS6/TAS3 (837 nts) & EEMJ-2003175 \\
\hline Hypnum subimponens & Bryopsida/Bryidae & Hypnales & TAS6/TAS3 (823 nts) & LNSF-2068452 \\
\hline Pseudotaxiphyllum elegans & Bryopsida/Bryidae & Hypnales & TAS6/TAS3 (1,590 nts) & QKQO-2009669 \\
\hline Anomodon attenuatus & Bryopsida/Bryidae & Hypnales & TAS6/TAS3 (843 nts) & QMWB-2059873 \\
\hline Anomodon rostratus & Bryopsida/Bryidae & Hypnales & TAS6/TAS3 (829 nts) & VBMM-2003482 \\
\hline Schwetschkeopsis fabronia & Bryopsida/Bryidae & Hypnales & TAS6/TAS3 (854 nts) & IGUH-2166854 \\
\hline Leucodon sciuroides & Bryopsida/Bryidae & Hypnales & TAS6/TAS3 (852 nts) & ZACW-2016434 \\
\hline Fontinalis antipyretica & Bryopsida/Bryidae & Hypnales & TAS6/TAS3 (1,410 nts) & DHWX-2007057 \\
\hline Rhytidiadelphus loreus & Bryopsida/Bryidae & Hypnales & TAS6/TAS3 (830 nts) & WSPM-2009782 \\
\hline Rhynchostegium serrulatum & Bryopsida/Bryidae & Hypnales & TAS6/TAS3 (853 nts) & JADL-2047695 \\
\hline Climacium dendroides & Bryopsida/Bryidae & Hypnales & TAS6/TAS3 (809 nts) & MIRS-2012325 \\
\hline Calliergon cordifolium & Bryopsida/Bryidae & Hypnales & TAS6 (95 nts) & TAVP-2006322 \\
\hline Neckera douglasii & Bryopsida/Bryidae & Hypnales & TAS6/TAS3 (839 nts) & TMAJ-2023603 \\
\hline Plagiomnium insigne & Bryopsida/Bryidae & Bryales & TAS6/TAS3 (914 nts) & BGXB-2010105 \\
\hline Orthotrichum lyellii & Bryopsida/Bryidae & Orthotrichales & TAS6 (192 nts) & CMEQ-2080784 \\
\hline Hedwigia ciliata & Bryopsida/Bryidae & Hedwigiales & TAS6/TAS3 (877 nts) & YWNF-2050742 \\
\hline Philonotis fontana & Bryopsida/Bryidae & Bartramiales & TAS6/TAS3 (893 nts) & ORKS-2058791 \\
\hline Aulacomnium heterostichum & Bryopsida/Bryidae & Rhizogoniales & TAS6/TAS3 (863 nts) & WNGH-2088134 \\
\hline Scouleria aquatic & Bryopsida/Dicranidae & Scouleriales & TAS6/TAS3 (partial) & BPSG-2088977 \\
\hline Syntrichia princeps & Bryopsida/Dicranidae & Pottiales & TAS6/TAS3 (partial) & GRKU-2074985 \\
\hline Leucobryum glaucum & Bryopsida/Dicranidae & Dicranales & TAS6/TAS3 (763 nts) & RGKI-2062694 \\
\hline Leucobryum albidum & Bryopsida/Dicranidae & Dicranales & TAS6/TAS3 (763 nts) & VMXJ-2128109 \\
\hline Dicranum scoparium & Bryopsida/Dicranidae & Dicranales & TAS6 (105 nts) & NGTD-2092412 \\
\hline Ceratodon purpureus & Bryopsida/Dicranidae & Pseudoditrichales & TAS6/TAS3 (1,121 nts) & $\begin{array}{l}\text { FFPD-2005850 } \\
\text { SRX2065999 }\end{array}$ \\
\hline Racomitrium varium & Bryopsida/Dicranidae & Grimmiales & TAS6/TAS3 (724 nts) & RDOO-2117129 \\
\hline Physcomitrium_sp. & Bryopsida/Funariidae & Funariales & TAS6 (partial) & YEPO-2071108 \\
\hline Physcomitrium_sp. & Bryopsida/Funariidae & Funariales & TAS6 (178 nts) & YEPO-2000016 \\
\hline Physcomitrium_sp. & Bryopsida/Funariidae & Funariales & TAS6/TAS3 (821 nts) & YEPO-2016361 \\
\hline Encalypta streptocarpa & Bryopsida/Funariidae & Encalyptales & TAS6/TAS3 (883 nts) & KEFD-2058811 \\
\hline Diphyscium foliosum & Bryopsida/Diphysciidae & Diphyscales & TAS6/TAS3 (832 nts) & AWOI-2069791 \\
\hline Tetraphis pellucida & Tetraphidopsida & Tetraphidales & TAS6 (partial) & HVBQ-2112923 \\
\hline Atrichum angustatum & Polytrichopsida & Polytrichales & TAS6/TAS3 (810 nts) & ZTHV-2082998 \\
\hline Andreaea rupestris & Andreaeopsida & Andreaeales & TAS6/TAS3 (869 nts) & WOGB-2010369 \\
\hline Takakia lepidozioides & Takakiopsida & Takakiales & TAS6/TAS3 (1,040 nts) & SKQD-2076588 \\
\hline
\end{tabular}

Notes.

${ }^{a}$ The length indicates total size of TAS6-TAS3 complex element (from the 5' miR529 target site in TAS6 to 3' miR390 target site in TAS3) or isolated TAS6 (between miR529 and miR156 target sites). 


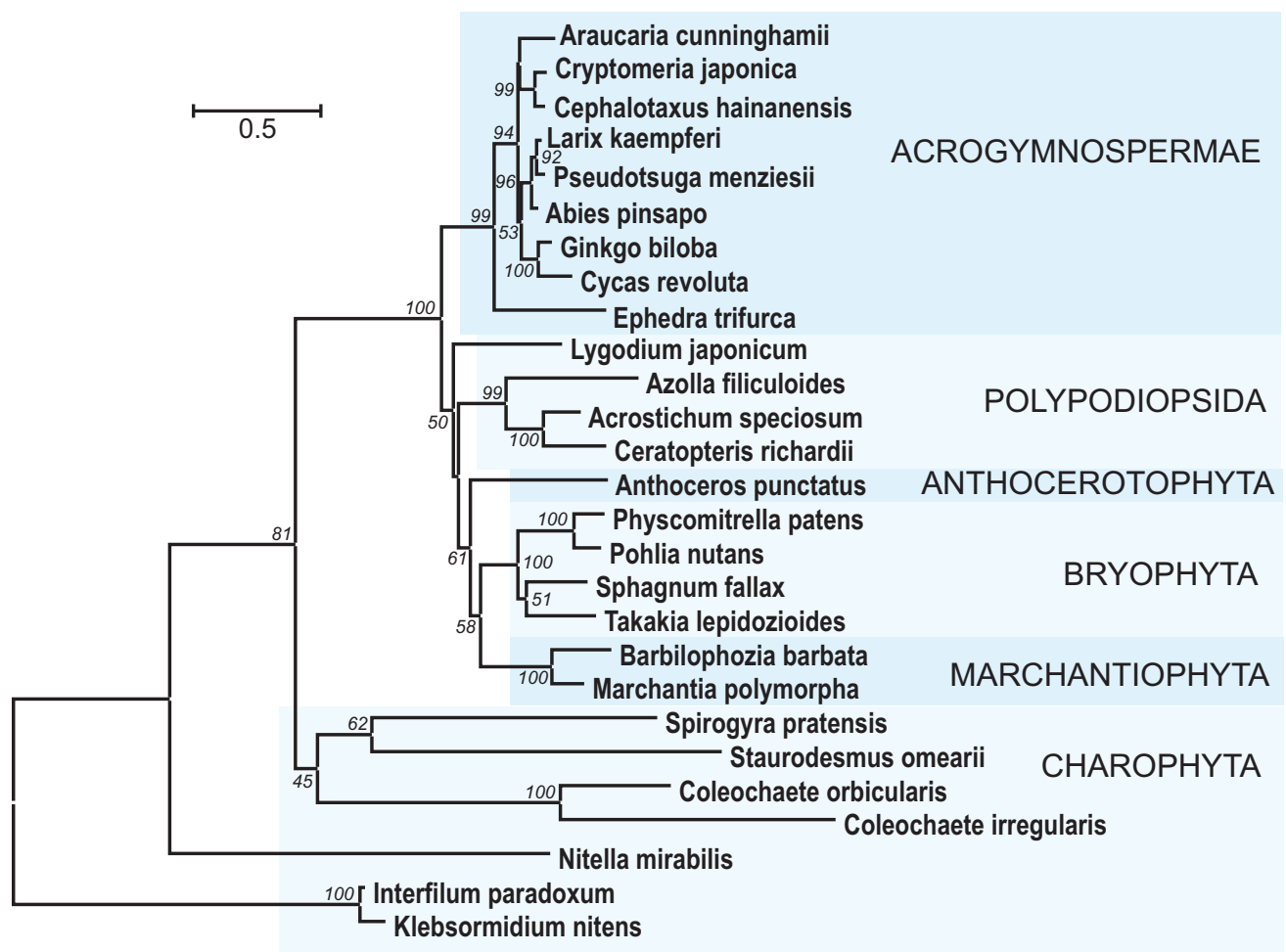

Figure 7 The phylogenetic tree constructed from conserved regions of SGS3 protein sequences from 27 selected streptophytes by the Neighbor-Joining method with 1000 bootstrap replications. There were a total of 419 positions in the final dataset. Evolutionary analyses were conducted in MEGA7 (Kumar, Stecher \& Tamura, 2016). Bootstrap support values $\geq 50 \%$ are shown. The evolutionary distances were computed using the JTT matrix-based method and are in the units of the number of amino acid substitutions per site. The rate variation among sites was modeled with a gamma distribution (shape parameter = 1). The tree was rooted at two Klebsormidiaceae charopytes. Accession numbers from NCBI ot PHYTOZOME data banks see in Fig. S5.

Full-size DOI: 10.7717/peerj.4636/fig-7

XS domain (Bateman, 2002; Zhang \& Trudeau, 2008). Importantly, the short N-terminal zf-XS domain is characteristic for functional SGS3 proteins, since the XS domain-containing protein of Selaginella moellendofii lacking TAS-generating machinery (Banks et al., 2011) possesses no zf-XS domain upstream of XS domain and instead contains the C-terminal RING zf region (see NCBI accession XP_002979112). However, it should be noted that the lack of TAS3 pathway and SGS3 is not universal for lycophytes (Xia, Xu \& Meyers, 2017).

In addition to class Bryopsida, SGS3 protein sequences were revealed for members of classes Marchantiopsida, Jungermanniopsida, Anthocerotopsida, Takakiopsida and Sphagnopsida (Fig. 7 and Fig. S5). Most importantly, search for the SGS3 coding sequences in transcriptomes of four charophyte classes (Klebsormidiophyceae) also revealed the SGS3like proteins in representatives of all these taxa (Fig. 7, Figs. S5, S6). This observation was in agreement with the fact that SGS3-like coding sequence was found in the fully sequenced and annotated genome of Klebsormidium nitens (NCBI accession GAQ92898) (Hori et al., 
2014). Moreover, the characteristic motifs of land plant SGS3 proteins (Bateman, 2002) were revealed in the protein sequences from charophyte algae (Figs. S5, S6).

Importantly, in the dendrogram based on comparisons of 27 aligned SGS3 protein sequences, the position of charophytes (Fig. 7) nearly corresponded to the commonly accepted Viridiplantae phylogenetic tree (Shaw, Szövényi \& Shaw, 2011; Delwiche \& Cooper, 2015; Harrison, 2017), where class Zygnemophyceae (Spirogyra pratensis) was a sister group for all land plants. It has become clear that evolving the SGS3-like genes was not directly connected to the appearance of TAS loci in Viridiplantae, since Chlorophyta species, lacking SGS3, encode not only critical enzyme machinery including DCLs, RDRs, and AGOs (You et al., 2017), but also PHAS loci (Zheng et al., 2015). Despite our extensive searches, no SGS3 genes could be identified also in brown and red algae, and this is in agreement with previously published data on green algae (Zheng et al., 2015).

\section{DISCUSSION}

Our current analyses revealed previously undiscovered TAS3 loci in bryophytes from classes Takakiopsida, Sphagnopsida and Anthocerotopsida. In Folioceros fuciformis (family Anthocerotaceae) we found a TAS3-like sequence which is obviously similar to Bryopsida class III TAS3 species (Fig. 3), whereas Takakiopsida TAS3 locus showed relatedness to class II of TAS3 with typical positioning of tasiAP2 and tasiARF-a2 sequences and no tasiARF-a3 sites (Xia, Xu \& Meyers, 2017). Unexpectedly, all predicted Sphagnopsida TAS3 loci showed no conventional internal structural organization of the moss class II and class III TAS3 species. Excepting miR390-targeting regions, the only recognizable conserved site was tasiARF-a2 sequence (Fig. 2).

It was shown that structural organization of Marchantiopsida and Jungermanniopsida TAS3 loci were quite similar, whereas Marchantiophyta TAS3 species were obviously different from those of Bryophyta. These loci were proposed to belong to TAS3 class I with species containing two conserved sequence blocks which presumably represent functional ta-siRNAs (Xia, Xu\& Meyers, 2017). One of these blocks was found in the vicinity of the $3^{\prime}$-terminal miR390 binding site and corresponded to Bryopsida tasi-AP2 sequence (Krasnikova et al., 2013), whereas another one (tasiARF-a1), unique among lower land plants, was located closer to the $5^{\prime}$-terminal miR390 binding site in Marchantiopsida and Jungermanniopsida TAS3 (Tsuzuki et al., 2016; Xia, Xu \& Meyers, 2017) (Fig. 5, Fig. S3).

Assuming occurrence of tasiARFs as potential products of TAS3 in all main lineages of land plants (Marchantiophyta, Anthocerotophyta and Bryophyta), recent paper proposed that the earliest function of TAS3 could contribute to the production of ta-siRNAs targeting ARF genes, and, since green algae encode no ARF genes, TAS3 likely appeared first in land plants (Xia, Xu\& Meyers, 2017). However, extensive comparative sequence analysis showed that charophyte algae representing the sister group to all land plants (colonized terrestrial environments approximately 480 million years ago, see for references Fischer (2018) and Puttick et al. (2018)) could also encode ARF-like proteins including all sequence domains typical for bryophyte and angiosperm ARFs (Mutte et al., 2017). Moreover, our current data showed that TAS3-like loci are encoded by the representatives of all main 
taxa among non-vascular plants. These observations suggest that the TAS3 evolution started in a common ancestor of land plants, likely belonging to a still unknown lineage of charophytes. Identification of the canonical motifs of land plant SGS3 in charophyte proteins (see above) indirectly supports this speculation. However, it should be kept in mind that evolving the SGS3-like genes could not be connected solely to the appearance of PHAS loci in Viridiplantae, since green algae and brown algae species were found to encode not only essential silencing machinery enzymes including DCLs, RDRs and AGOs, but also PHAS loci (Billoud et al., 2014; Zheng et al., 2015; Singh et al., 2015; Dueck et al., 2016; You et al., 2017; Cock et al., 2017). Finally, it can be proposed that the failure to identify charophyte TAS3 loci may be related to (i) the incompleteness of the available sequence data; (ii) evolving by charophytes the one-hit TAS3 genes (De Felippes et al., 2017); or (iii) the use of miRNA species with sequences other than land plant miR390 for TAS precursor processing.

\section{CONCLUSIONS}

The current data on the structural organization of TAS3-like loci in all main classes of land non-vascular plants reveal three types of TAS3 loci, namely, (i) Bryopsida-like TAS3 (classes II and III, Xia, Xu \& Meyers, 2017) found in Bryophyta plants (excepting Sphagnopsida) and Anthocerotopsida, (ii) Marchantiophyta-like TAS3 (class I, Xia, Xu \& Meyers, 2017) and (iii) Sphagnopsida-like TAS3 (this paper). Clearly recognizable common ta-siRNAs is represented in these TAS3 types by tasiARF sequences. Occurrence of primitive SGS3 and ARF genes in charophytes (Mutte et al., 2017 and this paper) supports the idea that TAS3-like genes first appeared in the hypothetical common precursor of land plants (Xia, Xu \& Meyers, 2017) to regulate auxin signaling (Leyser, 2018).

\section{ACKNOWLEDGEMENTS}

We thank researchers who contributed samples used in this study to the $1 \mathrm{KP}$ initiative.

\section{ADDITIONAL INFORMATION AND DECLARATIONS}

\section{Funding}

The work of Sergey Morozov, Tatiana Erokhina and Andrey Solovyev was supported by the Russian Science Foundation (grant 17-14-01032). The work of Irina Milyutina and Alexey Troitsky was supported by the Russian Foundation for Basic Research (grant 18-04-00574-a). The work of Liudmila Ozerova was supported by the State Assignment of MBG RAS on the base of the Unique Scientific Installation "The Fund Greenhouse". The funders had no role in study design, data collection and analysis, decision to publish, or preparation of the manuscript.

\section{Grant Disclosures}

The following grant information was disclosed by the authors:

Russian Science Foundation: 17-14-01032. 
Russian Foundation for Basic Research: 18-04-00574-a.

State Assignment of MBG RAS on the base of the Unique Scientific Installation "The Fund Greenhouse".

\section{Competing Interests}

The authors declare there are no competing interests.

\section{Author Contributions}

- Sergey Y. Morozov conceived and designed the experiments, analyzed the data, prepared figures and/or tables, authored or reviewed drafts of the paper, approved the final draft.

- Irina A. Milyutina conceived and designed the experiments, performed the experiments, contributed reagents/materials/analysis tools, approved the final draft.

- Tatiana N. Erokhina and Liudmila V. Ozerova performed the experiments, contributed reagents/materials/analysis tools, prepared figures and/or tables, approved the final draft.

- Alexey V. Troitsky and Andrey G. Solovyev conceived and designed the experiments, analyzed the data, authored or reviewed drafts of the paper, approved the final draft.

\section{DNA Deposition}

The following information was supplied regarding the deposition of DNA sequences:

New sequences described here are accessible via GenBank accession numbers MF682529 and MF682530.

\section{Supplemental Information}

Supplemental information for this article can be found online at http://dx.doi.org/10.7717/ peerj.4636\#supplemental-information.

\section{REFERENCES}

Alaba S, Piszczalka P, Pietrykowska H, Pacak AM, Sierocka I, Nuc PW, Singh K, Plewka P, Sulkowska A, Jarmolowski A, Karlowski WM, Szweykowska-Kulinska Z. 2015. The liverwort Pellia endiviifolia shares microtranscriptomic traits that are common to green algae and land plants. New Phytology 206:352-367

DOI 10.1111/nph.13220.

Allen E, Howell M. 2010. miRNAs in the biogenesis of trans-acting siRNAs in higher plants. Seminars in Cell and Developmental Biology 21:798-804 DOI 10.1016/j.semcdb.2010.03.008.

Allen E, Xie Z, Gustafson A, Carrington J. 2005. MicroRNA-directed phasing during trans-acting siRNA biogenesis in plants. Cell 121:207-221

DOI 10.1016/j.cell.2005.04.004.

Arif MA, Fattash I, Ma Z, Cho SH, Beike AK, Reski R, Axtell MJ, Frank W. 2012. DICER-LIKE3 activity in Physcomitrella patens DICER-LIKE4 mutants causes severe developmental dysfunction and sterility. Molecular Plant 5:1281-1294 DOI $10.1093 / \mathrm{mp} / \mathrm{sss} 036$. 
Arif MA, Frank W, Khraiwesh B. 2013. Role of RNA interference (RNAi) in the Moss Physcomitrella patens. International Journal of Molecular Sciences 14:1516-1540 DOI 10.3390/ijms14011516.

Axtell MJ. 2013. Classification and comparison of small RNAs from plants. Annual Review of Plant Biology 64:137-159 DOI 10.1146/annurev-arplant-050312-120043.

Axtell MJ, Meyers BC. 2018. Revisiting criteria for plant miRNA annotation in the era of big data. The Plant Cell 30:272-284 DOI 10.1105/tpc.17.00851.

Banks JA, Nishiyama T, Hasebe M, Bowman JL, Gribskov M, DePamphilis C, Albert VA, Aono N, Aoyama T, Ambrose BA, Ashton NW, Axtell MJ, Barker E, Barker MS, Bennetzen JL, Bonawitz ND, Chapple C, Cheng C, Correa LG, Dacre M, DeBarry J, Dreyer I, Elias M, Engstrom EM, Estelle M, Feng L, Finet C, Floyd SK, Frommer WB, Fujita T, Gramzow L, Gutensohn M, Harholt J, Hattori M, Heyl A, Hirai T, Hiwatashi Y, Ishikawa M, Iwata M, Karol KG, Koehler B, Kolukisaoglu U, Kubo M, Kurata T, Lalonde S, Li K, Li Y, Litt A, Lyons E, Manning G, Maruyama T, Michael TP, Mikami K, Miyazaki S, Morinaga S, Murata T, Mueller-Roeber B, Nelson DR, Obara M, Oguri Y, Olmstead RG, Onodera N, Petersen BL, Pils B, Prigge M, Rensing SA, Riao-Pachn DM, Roberts AW, Sato Y, Scheller HV, Schulz B, Schulz C, Shakirov EV, Shibagaki N, Shinohara N, Shippen DE, Sørensen I, Sotooka R, Sugimoto N, Sugita M, Sumikawa N, Tanurdzic M, Theissen G, Ulvskov P, Wakazuki S, Weng JK, Willats WW, Wipf D, Wolf PG, Yang L, Zimmer AD, Zhu Q, Mitros T, Hellsten U, Loqu D, Otillar R, Salamov A, Schmutz J, Shapiro H, Lindquist E, Lucas S, Rokhsar D, Grigoriev IV. 2011. The selaginella genome identifies genetic changes associated with the evolution of vascular plants. Science 332:960-963 DOI 10.1126/science.1203810.

Bateman A. 2002. The SGS3 protein involved in PTGS finds a family. BMC Bioinformatics 3:21 DOI 10.1186/1471-2105-3-21.

Billoud B, Nehr Z, Le Bail A, Charrier B. 2014. Computational prediction and experimental validation of microRNAs in the brown alga Ectocarpus siliculosus. Nucleic Acids Research 42:417-429 DOI 10.1093/nar/gkt856.

Bologna N, Voinnet O. 2014. The diversity, biogenesis, and activities of endogenous silencing small RNAs in Arabidopsis. Annual Review of Plant Biology 65:473-503 DOI 10.1146/annurev-arplant-050213-035728.

Borges F, Martienssen RA. 2015. The expanding world of small RNAs in plants. Nature Reviews Molecular Cell Biology 16:727-741 DOI 10.1038/nrm4085.

Bowman JL, Kohchi T, Yamato KT, Jenkins J, Shu S, Ishizaki K, Yamaoka S, Nishihama R, Nakamura Y, Berger F, Adam C, Aki SS, Althoff F, Araki T, Arteaga-Vazquez MA, Balasubrmanian S, Barry K, Bauer D, Boehm CR, Briginshaw L, CaballeroPerez J, Catarino B, Chen F, Chiyoda S, Chovatia M, Davies KM, Delmans M, Demura T, Dierschke T, Dolan L, Dorantes-Acosta AE, Eklund DM, Florent SN, Flores-Sandoval E, Fujiyama A, Fukuzawa H, Galik B, Grimanelli D, Grimwood J, Grossniklaus U, Hamada T, Haseloff J, Hetherington AJ, Higo A, Hirakawa Y, Hundley HN, Ikeda Y, Inoue K, Inoue SI, Ishida S, Jia Q, Kakita M, Kanazawa T, Kawai Y, Kawashima T, Kennedy M, Kinose K, Kinoshita T, Kohara Y, Koide E, 
Komatsu K, Kopischke S, Kubo M, Kyozuka J, Lagercrantz U, Lin SS, Lindquist E, Lipzen AM, Lu CW, De Luna E, Martienssen RA, Minamino N, Mizutani M, Mizutani M, Mochizuki N, Monte I, Mosher R, Nagasaki H, Nakagami H, Naramoto S, Nishitani K, Ohtani M, Okamoto T, Okumura M, Phillips J, Pollak B, Reinders A, Rvekamp M, Sano R, Sawa S, Schmid MW, Shirakawa M, Solano R, Spunde A, Suetsugu N, Sugano S, Sugiyama A, Sun R, Suzuki Y, Takenaka M, Takezawa D, Tomogane H, Tsuzuki M, Ueda T, Umeda M, Ward JM, Watanabe Y, Yazaki K, Yokoyama R, Yoshitake Y, Yotsui I, Zachgo S, Schmutz J. 2017. Insights into land plant evolution garnered from the marchantia polymorpha genome. Cell 171:287-304 DOI 10.1016/j.cell.2017.09.030.

Cho SH, Coruh C, Axtell MJ. 2012. miR156 and miR390 regulate tasiRNA accumulation and developmental timing in Physcomitrella patens. The Plant Cell 24:4837-4849 DOI 10.1105/tpc.112.103176.

Chorostecki U, Moro B, Rojas AML, Debernardi JM, Schapire AL, Notredame C, Palatnik JF. 2017. Evolutionary footprints reveal insights into plant microRNA biogenesis. The Plant Cell 29:1248-1261 DOI 10.1105/tpc.17.00272.

Cock JM, Liu F, Duan D, Bourdareau S, Lipinska AP, Coelho SM, Tarver JE. 2017. Rapid evolution of microRNA loci in the brown algae. Genome Biology and Evolution 9:740-749 DOI 10.1093/gbe/evx038.

Delwiche CF, Cooper ED. 2015. The evolutionary origin of terrestrial life. Current Biology 25:R899-R910 DOI 10.1016/j.cub.2015.08.029.

De Felippes FF, Marchais A, Sarazin A, Oberlin S, Voinnet O. 2017. A single miR390 targeting event is sufficient for triggering TAS3-tasiRNA biogenesis in Arabidopsis. Nucleic Acids Research 45:5539-5554 DOI 10.1093/nar/gkx119.

Deng P, Muhammad S, Cao M, Wu L. 2018. Biogenesis and regulatory hierarchy of phased small interfering RNAs in plants. Plant Biotechnology Journal DOI 10.1111/pbi.12882.

Dueck A, Evers M, Henz SR, Unger K, Eichner N, Merkl R, Berezikov E, Engelmann JC, Weigel D, Wenzl S, Meister G. 2016. Gene silencing pathways found in the green alga Volvox carteri reveal insights into evolution and origins of small RNA systems in plants. BMC Genomics 17:853 DOI 10.1186/s12864-016-3202-4.

Fei Q, Xia R, Meyers B. 2013. Phased, secondary, small interfering RNAs in posttranscriptional regulatory networks. The Plant Cell 25:2400-2415 DOI 10.1105/tpc.113.114652.

Fischer WW. 2018. Early plants and the rise of mud. Science 359:994-995 DOI 10.1126/science.aas9886.

Harrison CJ. 2017. Development and genetics in the evolution of land plant body plans. Philosophical Transactions of the Royal Society B: Biological Sciences 372:20150490 DOI 10.1098/rstb.2015.0490.

Hori K, Maruyama F, Fujisawa T, Togashi T, Yamamoto N, Seo M, Sato S, Yamada T, Mori H, Tajima N. 2014. Klebsormidium flaccidum genome reveals primary factors for plant terrestrial adaptation. Nature Communications 5:3978 DOI 10.1038/ncomms4978. 
Katoh K, Standley DM. 2014. MAFFT Multiple sequence alignment software version 7: improvements in performance and usability. Molecular Biology and Evolution 30:772-780 DOI 10.1093/molbev/mst010.

Komiya R. 2017. Biogenesis of diverse plant phasiRNAs involves an miRNA-trigger and Dicer-processing. Journal of Plant Research 130:17-23

DOI 10.1007/s10265-016-0878-0.

Krasnikova M, Goryunov D, Troitsky A, Solovyev A, Ozerova L, Morozov S. 2013. Peculiar evolutionary history of miR390-guided TAS3-like genes in land plants. Scientific World Journal 2013:924153 DOI 10.1155/2013/924153.

Krasnikova M, Milyutina I, Bobrova V, Ozerova L, Troitsky A, Solovyev A, Morozov S. 2011. Molecular diversity of mir390-guided trans-acting siRNA precursor genes in lower land plants: experimental approach and bioinformatics analysis. Sequencing 2011:703683 DOI 10.1155/2011/703683.

Krasnikova M, Milyutina I, Bobrova V, Troitsky A, Solovyev A, Morozov S. 2009. Novel miR390-dependent transacting siRNA precursors in plants revealed by a PCR-based experimental approach and database analysis. Journal of Biomedicine and Biotechnology 2011:952304 DOI 10.1155/2009/952304.

Kumar S, Stecher G, Tamura K. 2016. MEGA7: molecular evolutionary genetics analysis version 7.0 for bigger datasets. Molecular Biology and Evolution 33:1870-1874 DOI 10.1093/molbev/msw054.

Lemieux C, Otis C, Turmel M. 2016. Comparative chloroplast genome analyses of streptophyte green algae uncover major structural alterations in the klebsormidiophyceae, coleochaetophyceae and zygnematophyceae. Frontiers in Plant Science 7:697 DOI 10.3389/fpls.2016.00697.

Lewis LA, Mishler BD, Vilgalys R. 1997. Phylogenetic relationships of the liverworts (Hepaticae), a basal embryophyte lineage, inferred from nucleotide sequence data of the chloroplast gene rbcL. Molecular Phylogenetics and Evolution 7:377-393 DOI 10.1006/mpev.1996.0395.

Leyser O. 2018. Auxin signaling. Plant Physiology 176:465-479 DOI 10.1104/pp.17.00765. Lin PC, Lu CW, Shen BN, Lee GZ, Bowman JL, Arteaga-Vazquez MA, Liu LY, Hong SF, Lo CF, Su GM, Kohchi T, Ishizaki K, Zachgo S, Althoff F, Takenaka M, Yamato KT, Lin SS. 2016. Identification of miRNAs and their targets in the liverwort marchantia polymorpha by integrating RNA-seq and degradome analyses. Plant Cell Physiology 57:339-358 DOI 10.1093/pcp/pcw020.

Liu H, Yu H, Tang G, Huang T. 2018. Small but powerful: function of microRNAs in plant development. Plant Cell Reports 37:515-528 DOI 10.1007/s00299-017-2246-5.

Morea EG, Da Silva EM, E Silva GF, Valente GT, Barrera Rojas CH, Vincentz M, Nogueira FT. 2016. Functional and evolutionary analyses of the miR156 and miR529 families in land plants. BMC Plant Biology 16:40 DOI 10.1186/s12870-016-0716-5.

Mutte S, Kato H, Rothfels C, Melkonian M, Wong GK-S, Weijers D. 2017. Origin and evolution of the nuclear auxin response system. bioRxiv 220731 DOI $10.1101 / 220731$. 
Ozerova L, Krasnikova M, Troitsky A, Solovyev A, Morozov S. 2013. TAS3 genes for small ta-siARF RNAs in plants belonging to subtribe Senecioninae: occurrence of prematurely terminated RNA precursors. Molekuliarnaia Genetika, Mikrobiologiia I Virusologiia 28:79-84.

Plavskin Y, Nagashima A, Perroud PF, Hasebe M, Quatrano RS, Atwal GS, Timmermans MC. 2016. Ancient trans-acting siRNAs confer robustness and sensitivity onto the auxin response. Developmental Cell 36:276-289 DOI 10.1016/j.devcel.2016.01.010.

Puttick MN, Morris JL, Williams TA, Cox CJ, Edwards D, Kenrick P, Pressel S, Wellman CH, Schneider H, Pisani D, Donoghue PCJ. 2018. The interrelationships of land plants and the nature of the ancestral embryophyte. Current Biology 28:733-745 DOI 10.1016/j.cub.2018.01.063.

Qiu YL. 2008. Phylogeny and evolution of charophytic algae and land plants. Journal of Systematics and Evolution 46:287-306 DOI 10.3724/SP.J.1002.2008.08035.

Rogers K, Chen X. 2013. Biogenesis, turnover, and mode of action of plant microRNAs. The Plant Cell 25:2383-2399 DOI 10.1105/tpc.113.113159.

Rosato M, Kovařík A, Garilleti R, Rosselló JA. 2016. Conserved organisation of 45S rDNA sites and rDNA gene copy number among major clades of early land plants. PLOS ONE 11:e0162544 DOI 10.1371/journal.pone.0162544.

Ruhfel BR, Gitzendanner MA, Soltis PS, Soltis DE, Burleigh JG. 2014. From algae to angiosperms-inferring the phylogeny of green plants (Viridiplantae) from 360 plastid genomes. BMC Evolutionary Biology 14:23 DOI 10.1186/1471-2148-14-23.

Samigullin TK, Yacentyuk SP, Degtyaryeva GV, Valieho-Roman KM, Bobrova VK, Capesius I, Martin WF, Troitsky AV, Filin VR, Antonov AS. 2002. Paraphyly of bryophytes and close relationship of hornworts and vascular plants inferred from chloroplast rDNA spacers sequence analysis. Arctoa 11:31-43 DOI 10.15298/arctoa.11.05.

Santin F, Bhogale S, Fantino E, Grandellis C, Banerjee AK, Ulloa RM. 2017. Solanum tuberosum StCDPK1 is regulated by miR390 at the posttranscriptional level and phosphorylates the auxin efflux carrier StPIN4 in vitro, a potential downstream target in potato development. Physiologia Plantarum 159:244-261 DOI 10.1111/ppl.12517.

Shaw AJ, Cox CJ, Buck WR, Devos N, Buchanan AM, Cave L, Seppelt R, Shaw B, Larraín J, Andrus R, Greilhuber J, Temsch EM. 2010. Newly resolved relationships in an early land plant lineage: Bryophyta class Sphagnopsida (peat mosses). American Journal of Botany 97:1511-1531 DOI 10.3732/ajb.1000055.

Shaw AJ, Szövényi P, Shaw B. 2011. Bryophyte diversity and evolution: windows into the early evolution of land plants. American Journal of Botany 98:352-369 DOI 10.3732/ajb.1000316.

Shaw AJ, Devos N, Liu Y, Cox CJ, Goffinet B, Flatberg KI, Shaw B. 2016. Organellar phylogenomics of an emerging model system: Sphagnum (peatmoss). Annals of Botany 118:185-196 DOI 10.1093/aob/mcw086. 
Singh RK, Gase K, Baldwin IT, Pandey SP. 2015. Molecular evolution and diversification of the Argonaute family of proteins in plants. BMC Plant Biology 15:23 DOI 10.1186/s12870-014-0364-6.

Tsuzuki M, Nishihama R, Ishizaki K, Kurihara Y, Matsui M, Bowman JL, Kohchi T, Hamada T, Watanabe Y. 2016. Profiling and characterization of small rnas in the liverwort, marchantia polymorpha, belonging to the first diverged land plants. Plant Cell Physiology 57:359-372 DOI 10.1093/pcp/pcv182.

Wickett NJ, Mirarab S, Nguyen N, Warnow T, Carpenter E, Matasci N, Ayyampalayam S, Barker MS. 2014. Phylotranscriptomic analysis of the origin and early diversification of land plants. Proceedings of the National Academy of Sciences of the United States of America 111:E4859-E4868 DOI 10.1073/pnas.1323926111.

Xia R, Meyers BC, Liu Z, Beers EP, Ye S, Liu Z. 2013. MicroRNA superfamilies descended from miR390 and their roles in secondary small interfering RNA Biogenesis in Eudicots. The Plant Cell 25:1555-1572 DOI 10.1105/tpc.113.110957.

Xia R, Xu J, Meyers BC. 2017. The emergence, evolution, and diversification of the miR390 TAS3 ARF pathway in land plants. The Plant Cell 29:1232-1247 DOI 10.1105/tpc.17.00185.

Yoshikawa M. 2013. Biogenesis of trans-acting siRNAs, endogenous secondary siRNAs in plants. Genes \& Genetic Systems 88:77-84 DOI 10.1266/ggs.88.77.

You C, Cui J, Wang H, Qi X, Kuo L-Y, Ma H, Gao L, Mo B, Chen X. 2017. Conservation and divergence of smallRNA pathways and microRNAs in plants. Genome Biology 18:158 DOI 10.1186/s13059-017-1291-2.

Zhai J, Jeong D, De Paoli E, Park S, Rosen B, Li Y, González A, Yan Z, Kitto S, Grusak M, Jackson S, Stacey G, Cook D, Green P, Sherrier D, Meyers M. 2011. MicroRNAs as master regulators of the plant NB-LRR defense gene family via the production of phased, trans-acting siRNAs. Genes \& Development 25:2540-2553 DOI 10.1101/gad.177527.111.

Zhang D, Trudeau VL. 2008. The XS domain of a plant specific SGS3 protein adopts a unique RNA recognition motif (RRM) fold. Cell Cycle 7:2268-2270 DOI 10.4161/cc.7.14.6306.

Zheng Y, Wang Y, Wu J, Ding B, Fei Z. 2015. A dynamic evolutionary and functional landscape of plant phased small interfering RNAs. BMC Biology 13:32 DOI 10.1186/s12915-015-0142-4. 